\title{
Antenna subtraction at NNLO with hadronic initial states: double real initial-initial configurations
}

\author{
A. Gehrmann-De Ridder, ${ }^{a}$ T. Gehrmann ${ }^{b}$ and M. Ritzmann ${ }^{c}$ \\ ${ }^{a}$ Institute for Theoretical Physics, \\ ETH, CH-8093 Zürich, Switzerland \\ ${ }^{b}$ Institut für Theoretische Physik, Universität Zürich, \\ Winterthurerstrasse 190,CH-8057 Zürich, Switzerland \\ ${ }^{c}$ Institut de Physique Théorique, \\ CEA-Saclay, F-91191 Gif-sur-Yvette cedex, France \\ E-mail: gehra@itp.phys.ethz.ch, thomas.gehrmann@uzh.ch , \\ mathias.ritzmann@cea.fr
}

ABSTRACT: The antenna subtraction method handles real radiation contributions in higher order corrections to jet observables. The method is based on antenna functions, which encapsulate all unresolved radiation between a pair of hard radiator partons. To apply this method to compute hadron collider observables, initial-initial antenna functions with both radiators in the initial state are required in unintegrated and integrated forms. In view of extending the antenna subtraction method to next-to-next-to-leading order (NNLO) calculations at hadron colliders, we derive the full set of initial-initial double real radiation antenna functions in integrated form.

Keywords: Jets, Hadronic Colliders

ARXIV EPRINT: 1207.5779 


\section{Contents}

1 Introduction $\quad 1$

2 Initial-initial antenna subtraction at NNLO 3

3 Antenna functions for double real radiation 5

3.1 Definition of the antenna functions 5

$\begin{array}{lll}3.2 & \text { Phase space factorisation and mappings } & 7\end{array}$

4 Integration over the double real radiation phase space $\quad 9$

5 Results 11

$\begin{array}{lll}5.1 & \text { Quark-antiquark antenna functions } & 12\end{array}$

$\begin{array}{ll}5.2 \text { Quark-gluon antenna functions } & 13\end{array}$

$\begin{array}{ll}5.3 \text { Gluon-gluon antenna functions } & 15\end{array}$

6 Conclusions 16

$\begin{array}{ll}\text { A Master integrals } & \mathbf{1 7}\end{array}$

$\begin{array}{ll}\text { A.1 Hard region } & 19\end{array}$

$\begin{array}{lll}\text { A.2 Collinear } x_{1} \text { region } & 25\end{array}$

$\begin{array}{llr}\text { A.3 Collinear } x_{2} \text { region } & 29\end{array}$

$\begin{array}{lll}\text { A.4 Soft region } & 33\end{array}$

\section{Introduction}

Jet production at high energy colliders allows to reconstruct the parton-level dynamics of the hard interaction processes. Jet cross sections are therefore ideally suited for precision studies of QCD $[1,2]$ to determine the strong coupling constant or parton distribution functions. They can be measured to a very high experimental accuracy, which has to be met by an equally high theoretical precision in order to perform meaningful comparisons. This precision demands the inclusion of next-to-next-to-leading order (NNLO) QCD corrections.

NNLO calculations of observables with $n$ jets in the final state require several ingredients: the two-loop corrected $n$-parton matrix elements, the one-loop corrected $(n+1)$ parton matrix elements, and the tree-level $(n+2)$-parton matrix elements. For many massless jet observables of phenomenological interest, these matrix elements are available for some time already.

The $(n+1)$-parton and $(n+2)$-parton matrix elements contribute to $n$ jet observables at NNLO if the extra partons are unresolved or are clustered to form an $n$-jet final state. Consequently, these extra partons are unconstrained in the soft and collinear regions, and 
yield infrared divergences. In these cases, the infrared singular parts of the matrix elements need to be extracted and integrated over the phase space appropriate to the unresolved configuration to make the infrared pole structure explicit. The single soft and collinear limits of one-loop matrix elements [3-13] and the double unresolved limits of tree-level matrix elements [14-20] are process-independent, and result in a factorisation into an unresolved factor times a matrix element of lower multiplicity.

To determine the contribution to NNLO jet observables from these configurations, one has to find subtraction terms which coincide with the full matrix element in the unresolved limits and are still sufficiently simple to be integrated analytically in order to cancel their infrared pole structure with the virtual contributions. Often starting from systematic methods for subtraction at NLO [21-27], several NNLO subtraction methods have been proposed in the literature [28-45], and are worked out to a varying level of sophistication.

For observables with partons only in the final state, an NNLO subtraction formalism, antenna subtraction, has been derived in [46]. The antenna subtraction formalism constructs the subtraction terms from antenna functions. Each antenna function encapsulates all singular limits due to the emission of one or two unresolved partons between two colour-connected hard radiator partons. This construction exploits the universal factorisation of matrix elements and phase space in all unresolved limits. The antenna functions are derived systematically from physical matrix elements [47-49]. This formalism has been applied in the derivation of NNLO corrections to three-jet production in electron-positron annihilation [50-54] and related event shapes [55-59], which were used subsequently in precision determinations of the strong coupling constant [60-70]. The formalism can be extended to include massive fermions [71-74]. Antenna subtraction has also been used as the starting point for a parton shower algorithm [75-78], using the fact that antennae capture the unresolved limits of QCD amplitudes to achieve a leading-log resummation.

For processes with initial-state partons, antenna subtraction has been fully worked out only to NLO so far [79]. In this case, one encounters two new types of antenna functions, initial-final antenna functions with one radiator parton in the initial state, and initial-initial antenna functions with both radiator partons in the initial state. The framework for the construction of NNLO antenna subtraction terms involving one or two partons in the initial state has been set up in $[80,81]$ in the context of a proof-of-principle implementation of the contribution of the purely gluonic contributions to di-jet production at hadron colliders. The initial-final and initial-initial antenna functions appearing in the NNLO subtraction terms are obtained from crossing the final-final antennae. Their integration has to be performed over the appropriate phase space. In the case of the initial-final antennae, this has been accomplished in [82]. The initial-initial one-loop antenna functions were integrated in [83]. Partial results have been obtained for the integrated initial-initial double real radiation tree-level antenna functions in [84], where all antennae involving a secondary quark-antiquark pair were integrated. It is the aim of the present paper to complete the NNLO antenna subtraction scheme for hadron collider processes by integrating the remaining initial-initial double real radiation antenna functions.

Other approaches to perform NNLO calculations of exclusive observables with initial state partons are the use of sector decomposition (partly in combination with subtrac- 
tion) and a subtraction method based on the transverse momentum structure of the final state. The sector decomposition algorithm [85-88] analytically decomposes both phase space and loop integrals into their Laurent expansion in dimensional regularisation, and performs a subsequent numerical computation of the coefficients of this expansion. Using this formalism, NNLO results were obtained for Higgs production [45, 89-91] and vector boson production [92] at hadron colliders. Both reactions were equally computed independently [93-95] using an NNLO subtraction formalism exploiting the specific transverse momentum structure of these observables [39], which was also applied to compute NNLO corrections to associated $W H$ production [96] and photon pair production [97]. A very promising approach could be the combination of subtraction with sector decomposition [40-42], which was recently applied in the calculation of NNLO corrections to the quark-induced processes in top quark pair production [98, 99].

This paper is structured as follows: in section 2, we briefly summarise the application of the antenna subtraction formalism to hadronic collisions, which was developed in [7981, 84]. The antenna functions required for double real radiation and their associated phase space mappings are described in section 3 while the phase space integration is described in section 4. The resulting integrated initial-initial antenna functions being too lengthy to be presented here, only their leading pole parts are given in section 5 . The full results are attached separately to this paper as a FORM $[100,101]$ file. Section 6 contains our conclusions and an outlook. The newly derived phase space master integrals required for our calculation are collected in the appendix.

\section{Initial-initial antenna subtraction at NNLO}

The partonic double real contribution to an NNLO $m$-jet cross section reads

$$
\begin{aligned}
\mathrm{d} \hat{\sigma}_{N N L O}^{R R}= & \mathcal{N}_{N N L O}^{R R} \sum_{\text {perms }} \mathrm{d} \Phi_{m+2}\left(k_{1}, \ldots, k_{m+2} ; p_{1}, p_{2}\right) \frac{1}{S_{m+2}} \\
& \times\left|\mathcal{M}_{m+2}\left(p_{1}, p_{2} ; k_{1}, \ldots, k_{m+2}\right)\right|^{2} J_{m}^{(m+2)}\left(k_{1}, \ldots, k_{m+2} ; p_{1}, p_{2}\right)
\end{aligned}
$$

In this equation, $\left|\mathcal{M}_{m+2}\left(p_{1}, p_{2} ; k_{1}, \ldots, k_{m+2}\right)\right|^{2}$ stands for the squared tree-level matrix element for the production of $(m+2)$ partons. This squared matrix element is decomposed in a colour-ordered manner into leading and subleading colour contributions (as for example derived explicitly in $[50,51])$. At leading colour, $\left|\mathcal{M}_{m+2}\right|^{2}$ consists of the squares of the colour-ordered amplitudes, while at subleading colour, it is made from the appropriate sum of interference terms of colour-ordered amplitudes. Both at leading and subleading colour, $\left|\mathcal{M}_{m+2}\right|^{2}$ can be decomposed such that each potentially unresolved parton is colourconnected only to two other partons. In the antenna subtraction approach, the construction of the subtraction terms follows this colour-ordered decomposition. The symmetry factor $S_{m+2}$ accounts for identical partons in the final state and $\sum_{\text {perms }}$ denotes the sum over all configurations with $m+2$ partons. The next-to-next-to leading order normalisation factor $\mathcal{N}_{N N L O}^{R R}$ includes all QCD-independent factors as well as the dependence on the renormalised QCD coupling constant $\alpha_{s}$. It is related to the normalisation factor present 
at leading order, $\mathcal{N}_{L O}$, which depends on the specific process and parton channel under consideration by,

$$
\mathcal{N}_{N N L O}^{R R}=\mathcal{N}_{L O}\left(\frac{\alpha_{s} N}{2 \pi}\right)^{2} \frac{\bar{C}(\epsilon)^{2}}{C(\epsilon)^{2}}
$$

where,

$$
\begin{aligned}
C(\epsilon) & =(4 \pi)^{\epsilon} \frac{e^{-\epsilon \gamma}}{8 \pi^{2}}, \\
\bar{C}(\epsilon) & =(4 \pi)^{\epsilon} e^{-\epsilon \gamma} .
\end{aligned}
$$

The initial state momenta are labelled as usual as $p_{1}$ and $p_{2}$ whereas the $m+2$ momenta in the final state are labelled $k_{1}, \ldots, k_{m+2}$. The $2 \rightarrow m+2$ particle phase space is denoted as

$$
\mathrm{d} \Phi_{m+2}\left(k_{1}, \ldots, k_{m+2} ; p_{1}, p_{2}\right)=\left[\mathrm{d} k_{1}\right] \ldots\left[\mathrm{d} k_{m+2}\right](2 \pi)^{d} \delta^{d}\left(p_{1}+p_{2}-k_{1}-\ldots-k_{m+2}\right)
$$

where we have introduced the abbreviation $\left[\mathrm{d} k_{l}\right]=\mathrm{d}^{d} k_{l} \delta^{+}\left(k_{l}^{2}\right) /(2 \pi)^{d-1}$. The jet function $J_{m}^{(m+2)}\left(k_{1}, \ldots, k_{m+2} ; p_{1}, p_{2}\right)$ ensures that out of $(m+2)$ final state partons, an observable with $m$ jets is built. The incoming parton momenta $p_{1}, p_{2}$ serve as reference directions to define transverse momenta and rapidities of the jets.

The real radiation contribution (2.1) contains infrared divergencies, which arise when one or two of the final state partons become unresolved. The numerical phase space integration in (2.1) can therefore not be carried out without prior introduction of a regulator or a subtraction. A subtraction term $\mathrm{d} \hat{\sigma}_{N N L O}^{S}$ is defined on the same phase space as $\mathrm{d} \hat{\sigma}_{N N L O}^{R R}$, and it approaches its integrand in all unresolved limits. Consequently $\mathrm{d} \hat{\sigma}_{N N L O}^{R R}-$ $\mathrm{d} \hat{\sigma}_{N N L O}^{S}$ is finite and the integration can be performed numerically. $\mathrm{d} \hat{\sigma}_{N N L O}^{S}$ is integrated over those parts of the phase space that contain infrared singularities and then combined with the virtual NNLO and the mass factorisation counterterm contributions to achieve the cancellation of infrared divergencies.

The double real radiation contribution $\mathrm{d} \hat{\sigma}_{N N L O}^{R R}$ can become singular if either one or two final state partons are unresolved (soft or collinear). Consequently, when constructing the corresponding subtraction term $\mathrm{d} \hat{\sigma}_{N N L O}^{S}$ in the antenna subtraction method, we must distinguish the following configurations according to the colour connection of the unresolved partons:

(a) One unresolved parton but the experimental observable selects only $m$ jets.

(b) Two colour-connected unresolved partons (colour-connected).

(c) Two unresolved partons that are not colour connected but share a common radiator (almost colour-unconnected).

(d) Two unresolved partons that are well separated from each other in the colour chain (colour-unconnected).

(e) Large angle soft gluon radiation. 
All cases except (b) and (e) can be accounted for by combinations of three-parton antenna functions, which were derived and integrated already in the context of antenna subtraction at NLO. Case (e) can always be described [81] by soft antenna functions in final-final kinematics, that were derived in [50-54]. In case (b), tree-level four-parton antennae are needed. The corresponding subtraction term for both radiators in the initial state reads:

$$
\begin{aligned}
& \mathrm{d} \hat{\sigma}_{N N L O}^{S, b,(i i)}=\mathcal{N}_{N N L O}^{R R} \sum_{m+2} \mathrm{~d} \Phi_{m+2}\left(k_{1}, \ldots, k_{m+2} ; p_{1}, p_{2}\right) \frac{1}{S_{m+2}} \\
& \quad \times \sum_{i l} \sum_{j k}\left(X_{i l, j k}^{0}-X_{l, j k}^{0} X_{i L, K}^{0}-X_{i, k j}^{0} X_{I l, J}^{0}\right)\left|\mathcal{M}_{m}\left(x_{i} p_{i}, x_{l} p_{l} ; \tilde{k}_{1}, \ldots, \tilde{k}_{i}, \tilde{k}_{l}, \ldots, \tilde{k}_{m+2}\right)\right|^{2} \\
& \quad \times J_{m}^{(m)}\left(\tilde{k}_{1}, \ldots, \tilde{k}_{i}, \tilde{k}_{l}, \ldots, \tilde{k}_{m+2} ; x_{i} p_{i}, x_{l} p_{l}\right) .
\end{aligned}
$$

The sum runs over all colour-adjacent pairs $j, k$ and implies that the hard momenta $i, l$ are chosen accordingly. Tree-level antenna functions are generically denoted $X^{0}$, we will give the precise definition in section 2. $X_{i l, j k}^{0}$ contains all the unresolved limits associated with $j$ and/or $k$ becoming becoming unresolved between the initial-state emitters $i$ and $l$. Its single unresolved limits are subtracted by products of three-particle antenna functions, ensuring that $\mathrm{d} \hat{\sigma}_{N N L O}^{S, b,(i i)}$ is only active in the double unresolved region. The reduced matrix element $\mathcal{M}$ is evaluated with a set of $m$ on-shell momenta which are obtained from the original ones by rescaling the initial-state momenta $p_{i}$ and $p_{l}$, omitting the final-state momenta $k_{j}$ and $k_{k}$ and applying a Lorentz boost to the remaining final-state momenta [79].

To analytically integrate the subtraction term (2.6), we employ dimensional regularisation in $d=4-2 \epsilon$ dimensions. We use the factorisation of the phase space

$$
\begin{aligned}
\mathrm{d} \Phi_{m+2}\left(k_{1}, \ldots, k_{m+2} ; p_{1}, p_{2}\right)= & \mathrm{d} \Phi_{m}\left(\tilde{k}_{1}, \ldots, \tilde{k}_{i}, \tilde{k}_{l}, \ldots, \tilde{k}_{m+2} ; x_{1} p_{1}, x_{2} p_{2}\right) \\
& \times \delta\left(x_{1}-\hat{x}_{1}\right) \delta\left(x_{2}-\hat{x}_{2}\right)\left[\mathrm{d} k_{j}\right]\left[\mathrm{d} k_{k}\right] \mathrm{d} x_{1} \mathrm{~d} x_{2},
\end{aligned}
$$

where $\hat{x}_{1}$ and $\hat{x}_{2}$ are defined throughout the phase space. In the case of collinear initial state radiation, they are identified with the collinear momentum fractions of the partons entering the hard scattering process.

The kinematics of the reduced matrix element appearing in (2.6) depends on the mapped momenta, and thus consequently on $q^{2}, x_{1}, x_{2}$. The integration of the antenna functions must therefore retain the dependence on $x_{1}, x_{2}$. The integrated initial-initial antenna functions are defined as:

$$
\mathcal{X}_{i l, j k}^{0}\left(x_{i}, x_{l}, \epsilon\right)=\frac{1}{[C(\epsilon)]^{2}} \int\left[\mathrm{d} k_{j}\right]\left[\mathrm{d} k_{k}\right] x_{i} x_{l} \delta\left(x_{i}-\hat{x}_{i}\right) \delta\left(x_{l}-\hat{x}_{l}\right) X_{i l, j k}^{0},
$$

where $C(\epsilon)$ is given in eq. (2.3).

\section{Antenna functions for double real radiation}

\subsection{Definition of the antenna functions}

Antenna functions are characterised by their parton content and their radiators, i.e. the pair of hard partons which they collapse to in the unresolved limits. Accordingly, we group 


\begin{tabular}{|ll|}
\hline \multicolumn{2}{|l|}{ quark-antiquark antennae } \\
\hline$A_{3}^{0} \quad A_{3}^{0}(\widehat{q}, \widehat{g}, \bar{q}), A_{3}^{0}(\widehat{q}, g, \widehat{\bar{q}})$ \\
\hline quark-gluon antennae \\
\hline$D_{3}^{0} \quad D_{3}^{0}(\widehat{q}, \widehat{g}, g), D_{3}^{0}(q, \widehat{g}, \widehat{g})$ \\
$E_{3}^{0} \quad E_{3}^{0}\left(\widehat{q}, \widehat{q^{\prime}}, \bar{q}^{\prime}\right), E_{3}^{0}\left(q, \widehat{q^{\prime}}, \widehat{\bar{q}^{\prime}}\right)^{* *}$ \\
\hline gluon-gluon antennae \\
\hline$F_{3}^{0} \quad F_{3}^{0}(\widehat{g}, g, \widehat{g})$ \\
$G_{3}^{0} \quad G_{3}^{0}(\widehat{g}, q, \widehat{\bar{q}}), G_{3}^{0}(g, \widehat{q}, \widehat{\bar{q}})^{* *}$ \\
\hline
\end{tabular}

Table 1. The tree-level three-particle initial-initial antennae and their distinct crossings. $D_{3}^{0}$ is symmetric under the interchange of the two gluons, $F_{3}^{0}$ under cyclic interchange of its arguments.

them into quark-antiquark, quark-gluon and gluon-gluon antennae. They are derived from physical matrix elements associated to the decay of a colourless particle into partons [4749]. The tree-level antenna functions are obtained by normalising the three- and fourparton tree-level colour sub-amplitudes squared to that of the basic two-parton process: The final-final three- and four-particle antennae are respectively defined by:

$$
\begin{gathered}
X_{i j k}^{0}=S_{i j k, I K} \frac{\left|\mathcal{M}_{i j k}^{0}\right|^{2}}{\left|\mathcal{M}_{I K}^{0}\right|^{2}}, \\
X_{i j k l}^{0}=S_{i j k l, I L} \frac{\left|\mathcal{M}_{i j k l}^{0}\right|^{2}}{\left|\mathcal{M}_{I L}^{0}\right|^{2}} .
\end{gathered}
$$

where $S$ denotes the symmetry factor associated with the antenna, which accounts both for potential identical particle symmetries and for the presence of more than one antenna in the basic two-parton process. It is chosen such that the antenna function reproduces the unresolved limits of a matrix element with identified particles. The initial-initial tree-level three- and four-parton antennae denoted by $X_{i k, l}^{0}$ and $X_{i l, j k}^{0}$ are obtained by crossing two partons to the initial state, starting from the final-final antennae. The distinct crossings of the three-parton antenna functions are listed in table 1. Crossings which are free of unresolved limits are marked with a ${ }^{* *}$.

The four-particle tree-level antenna functions are not determined by the species of the particles alone but also by the colour-connection. We distinguish leading-colour antennae, denoted by letters without tilde, where the particles are colour-connected in the order they are listed and subleading colour antennae, denoted by letters with tilde, where the gluons are photon-like. This notation has been established in [46, 82]. The unresolved limits of the initial-initial antennae can be obtained from those of the final-final antennae by crossing. The crossing of the triple-collinear splitting functions is explained in [102].

Any two particles of a four-particle final-final antenna can be crossed to the initial state to obtain an initial-initial antenna; therefore one final-final four-particle antenna gives rise to six initial-initial antennae. Due to symmetries, at most four of these initial-initial 


\begin{tabular}{|ll|}
\hline \multicolumn{2}{|l|}{ quark-antiquark antennae } \\
\hline$A_{4}^{0}$ & $A_{4}^{0}(\widehat{q}, g, g, \widehat{\bar{q}}), A_{4}^{0}(\widehat{q}, \widehat{g}, g, \bar{q}), A_{4}^{0}(\widehat{q}, g, \widehat{g}, \bar{q}), A_{4}^{0}(q, \widehat{g}, \widehat{g}, \bar{q})$ \\
$\widetilde{A}_{4}^{0}$ & $\widetilde{A}_{4}^{0}(\widehat{q}, g, g, \widehat{\bar{q}}), \widetilde{A}_{4}^{0}(\widehat{q}, \widehat{g}, g, \bar{q}), \widetilde{A}_{4}^{0}(q, \widehat{g}, \widehat{g}, \bar{q})$ \\
$B_{4}^{0}$ & $B_{4}^{0}\left(\widehat{q}, q^{\prime}, \bar{q}^{\prime}, \widehat{\bar{q}}\right), B_{4}^{0}\left(\widehat{q}, \widehat{q^{\prime}}, \bar{q}^{\prime}, \bar{q}\right), B_{4}^{0}\left(q, \widehat{q^{\prime}}, \widehat{\bar{q}}^{\prime}, \bar{q}\right)^{* *}$ \\
$C_{4}^{0}$ & $C_{4}^{0}(\widehat{q}, \bar{q}, q, \widehat{\bar{q}}), C_{4}^{0}(\widehat{q}, \bar{q}, \widehat{q}, \bar{q}), C_{4}^{0}(q, \widehat{\bar{q}}, \widehat{q}, \bar{q})^{* *}, C_{4}^{0}(q, \widehat{\bar{q}}, q, \widehat{\bar{q}})^{* *}$ \\
\hline
\end{tabular}

Table 2. The tree-level four-particle quark-antiquark antennae and their distinct crossings. $\widetilde{A}_{4}^{0}$ is symmetric under the interchange of the two photon-like gluons. The nonidentical-flavour antenna $B_{4}^{0}$ is separately symmetric under interchange of $q^{\prime}$ with $\bar{q}^{\prime}$ and of $q$ with $\bar{q}$.

\begin{tabular}{|ll|}
\hline \multicolumn{2}{|l|}{ quark-gluon antennae } \\
\hline$D_{4}^{0}$ & $D_{4}^{0}(\widehat{q}, \widehat{g}, g, g), D_{4}^{0}(\widehat{q}, g, \widehat{g}, g), D_{4}^{0}(q, \widehat{g}, \widehat{g}, g), D_{4}^{0}(q, \widehat{g}, g, \widehat{g})$ \\
$E_{4}^{0}$ & $E_{4}^{0}\left(\widehat{q}, \widehat{q^{\prime}}, \bar{q}^{\prime}, g\right), E_{4}^{0}\left(\widehat{q}, q^{\prime}, \bar{q}^{\prime}, \widehat{g}\right), E_{4}^{0}\left(q, \widehat{q^{\prime}}, \widehat{\bar{q}}^{\prime}, g\right), E_{4}^{0}\left(q, \widehat{q^{\prime}}, \bar{q}^{\prime}, \widehat{g}\right)$ \\
$\widetilde{E}_{4}^{0}$ & $\widetilde{E}_{4}^{0}\left(\widehat{q}, \widehat{q^{\prime}}, \bar{q}^{\prime}, g\right), \widetilde{E}_{4}^{0}\left(\widehat{q}, q^{\prime}, \bar{q}^{\prime}, \widehat{g}\right), \widetilde{E}_{4}^{0}\left(q, \widehat{q}^{\prime}, \widehat{\bar{q}}^{\prime}, g\right), \widetilde{E}_{4}^{0}\left(q, \widehat{q}^{\prime}, \bar{q}^{\prime}, \widehat{g}\right)$ \\
\hline
\end{tabular}

Table 3. The tree-level four-particle quark-gluon antennae and their distinct crossings. Due to the cyclic colour connection, $D_{4}^{0}$ is symmetric under interchange of the second and fourth gluon.

\begin{tabular}{|ll|}
\hline \multicolumn{2}{|l|}{ gluon-gluon antennae } \\
\hline$F_{4}^{0}$ & $F_{4}^{0}(\widehat{g}, \widehat{g}, g, g), F_{4}^{0}(\widehat{g}, g, \widehat{g}, g)$ \\
$G_{4}^{0}$ & $G_{4}^{0}(\widehat{g}, q, \bar{q}, \widehat{g}), G_{4}^{0}(\widehat{g}, \widehat{q}, \bar{q}, g), G_{4}^{0}(\widehat{g}, q, \widehat{\bar{q}}, g), G_{4}^{0}(g, \widehat{q}, \widehat{\bar{q}}, g)$ \\
$\widetilde{G}_{4}^{0}$ & $\widetilde{G}_{4}^{0}(\widehat{g}, q, \bar{q}, \widehat{g}), \widetilde{G}_{4}^{0}(\widehat{g}, \widehat{q}, \bar{q}, g), \widetilde{G}_{4}^{0}(g, \widehat{q}, \widehat{\bar{q}}, g)$ \\
$H_{4}^{0}$ & $H_{4}^{0}\left(\widehat{q}, \widehat{\bar{q}}, q^{\prime}, \bar{q}^{\prime}\right), H_{4}^{0}\left(\widehat{q}, \bar{q}, \widehat{q^{\prime}}, \bar{q}^{\prime}\right)$ \\
\hline
\end{tabular}

Table 4. The tree-level four-particle gluon-gluon antennae and their distinct crossings. $F_{4}^{0}$ is symmetric under cyclic interchange of its arguments. $\widetilde{G}_{4}^{0}$ is symmetric under the interchange of the two photon-like gluons as well as under the interchange of $q$ with $\bar{q}$. $H_{4}^{0}$ has three symmetries, $q \leftrightarrow \bar{q}, q^{\prime} \leftrightarrow \bar{q}^{\prime}$ and the flavour renaming $q \leftrightarrow q^{\prime}, \bar{q} \leftrightarrow \bar{q}^{\prime}$.

antennae are different. The independent crossings [84] are listed in tables $2-4$. To make the colour connection clear, in this list we write out the arguments of the antennae explicitly, i.e we write $X_{4}^{0}(\widehat{\imath}, j, \widehat{k}, l)$ (where $\widehat{\imath}$ denotes an incoming particle) instead of $X_{i k, j l}^{0}$. The initial-initial antennae which are free of singular limits are not needed for the construction of subtraction terms, but their integrated form could prove useful for cross-checks.

\subsection{Phase space factorisation and mappings}

The construction of subtraction terms requires a mapping from the original set of momenta onto a reduced set. The mapping interpolates between the different soft and collinear limits which the subtraction term regulates. An appropriate mapping for the initial-initial case, both for single and double unresolved configurations, has been discussed in [79]. By 
requiring momentum conservation and phase space factorisation, the phase space mapping is strongly constrained. The remapping of initial state momenta can only be a rescaling, since any transversal component would spoil the phase space factorisation. For two unresolved partons $j$ and $k$, a complete factorisation of the phase space into a convolution of an $m$-particle phase space depending on redefined momenta only and the phase space of the unresolved partons $j$ and $k$ can be achieved with a Lorentz boost. This boost maps the momentum $q=p_{1}+p_{2}-k_{j}-k_{k}$, with $q^{2}>0$ and $p_{1}, p_{2}$ being the momenta of the hard emitters, into the momentum $\tilde{q}=\hat{x}_{1} p_{1}+\hat{x}_{2} p_{2}$, where $\hat{x}_{1}$ and $\hat{x}_{2}$ are fixed in terms of the invariants as follows:

$$
\hat{x}_{1}=\left(\frac{s_{12}-s_{j 2}-s_{k 2}}{s_{12}-s_{1 j}-s_{1 k}} \frac{q^{2}}{s_{12}}\right)^{\frac{1}{2}}, \quad \hat{x}_{2}=\left(\frac{s_{12}-s_{1 j}-s_{1 k}}{s_{12}-s_{j 2}-s_{k 2}} \frac{q^{2}}{s_{12}}\right)^{\frac{1}{2}}
$$

These two definitions guarantee the overall momentum conservation in the mapped momenta and the correct soft and collinear behaviours. The two momentum fractions $\hat{x}_{1}$ and $\hat{x}_{2}$ satisfy the following limits in double unresolved configurations:

1. $j$ and $k$ soft: $\hat{x}_{1} \rightarrow 1, \hat{x}_{2} \rightarrow 1$,

2. $j$ soft and $k_{k}=z_{1} p_{1}: x_{1} \rightarrow 1-z_{1}, x_{2} \rightarrow 1$,

3. $k_{j}=z_{1} p_{1}$ and $k_{k}=z_{2} p_{2}: \hat{x}_{1} \rightarrow 1-z_{1}, \hat{x}_{2} \rightarrow 1-z_{2}$,

4. $k_{j}=z_{1} p_{1}, k_{k}=z_{2} p_{1}: \hat{x}_{1} \rightarrow 1-z_{1}-z_{2}, x_{2} \rightarrow 1$,

and all the limits obtained from the ones above by the exchange of $p_{1}$ with $p_{2}$ and of $k_{j}$ with $k_{k}$. The construction of NNLO antenna subtraction terms also requires that all single unresolved limits of the four-parton initial-initial antenna functions $X_{i l, j k}$, with radiators $i$ and $l$, have to be subtracted, such that the resulting subtraction term is active only in its double unresolved limits. A systematic subtraction of these single unresolved limits through products of two three-parton antenna functions can be performed only if the NNLO phase space mapping turns into an NLO phase space mapping in its single unresolved limits. A detailed discussion of the corresponding translation between these two momentum mappings can be found in [79].

The factorisation of the $(m+2)$-parton phase space into an $m$-parton phase space and an antenna phase space involving the unresolved partons $j$ and $k$ given in eq. (2.7) can equivalently be written as

$$
\begin{aligned}
\mathrm{d} \Phi_{m+2}\left(k_{1}, \ldots, k_{m+2} ; p_{1}, p_{2}\right)= & \mathrm{d} \Phi_{m}\left(\tilde{k}_{1}, \ldots, \tilde{k}_{i}, \tilde{k}_{l}, \ldots, \tilde{k}_{m+2} ; x_{1} p_{1}, x_{2} p_{2}\right) \\
& \times \mathcal{J} \delta\left(q^{2}-x_{1} x_{2} s_{12}\right) \delta\left(2\left(x_{2} p_{2}-x_{1} p_{1}\right) \cdot q\right) \\
& \times\left[\mathrm{d} k_{j}\right]\left[\mathrm{d} k_{k}\right] \mathrm{d} x_{1} \mathrm{~d} x_{2},
\end{aligned}
$$

where $\mathcal{J}$ is the Jacobian factor defined by

$$
\mathcal{J}=s_{12}\left(x_{1}\left(s_{12}-s_{1 j}-s_{1 k}\right)+x_{2}\left(s_{12}-s_{2 j}-s_{2 k}\right)\right) .
$$


Using this phase space parametrisation, we can express the integrated initial-initial antenna functions (2.8) as:

$$
\mathcal{X}_{i l, j k}^{0}\left(x_{1}, x_{2}, \epsilon\right)=\frac{1}{[C(\epsilon)]^{2}} \int\left[\mathrm{d} k_{j}\right]\left[\mathrm{d} k_{k}\right] \mathcal{J} x_{1} x_{2} \delta\left(C_{1}\right) \delta\left(C_{2}\right) X_{i l, j k}^{0},
$$

where

$$
\begin{aligned}
& C_{1}=q^{2}-x_{1} x_{2} s_{12}, \\
& C_{2}=2\left(x_{2} p_{2}-x_{1} p_{1}\right) \cdot q .
\end{aligned}
$$

In the following section, we describe how the integrals (3.4) are carried out by means of a reduction to phase space master integrals with constraints (3.5) and a derivation of the master integrals from their differential equations.

\section{Integration over the double real radiation phase space}

The initial-initial antenna functions have the scattering kinematics

$$
p_{1}+p_{2} \rightarrow k_{j}+k_{k}+q
$$

where $q$ is the momentum of the outgoing colourless particle. The momenta satisfy:

$$
p_{1}^{2}=p_{2}^{2}=0, \quad k_{j}^{2}=k_{k}^{2}=0, \quad q^{2}=\tilde{q}^{2}=x_{1} x_{2} s_{12} .
$$

The four-parton initial-initial antennae need to be integrated over the phase space of the unresolved partons $j$ and $k$. This integration yields a result which depends only on $q^{2}, x_{1}$ and $x_{2}$. The dependence on $q^{2}$ is only multiplicative, according to the mass dimension of the integral.

Using the optical theorem, these phase space integrals are expressed as cuts of forward scattering two-loop diagrams, where the $\delta$-function conditions fixing $x_{1}$ and $x_{2}$ are introduced through non-standard propagators $[103,104]$. The full set of propagators appearing in the four-parton antenna functions is:

$$
\begin{aligned}
D_{j 1} & =\left(p_{1}-k_{j}\right)^{2}, \\
D_{k 1} & =\left(p_{1}-k_{k}\right)^{2}, \\
D_{j 2} & =\left(p_{2}-k_{j}\right)^{2}, \\
D_{k 2} & =\left(p_{2}-k_{k}\right)^{2}, \\
D_{j k} & =\left(k_{j}+k_{k}\right)^{2}, \\
D_{j k 1} & =\left(p_{1}-k_{j}-k_{k}\right)^{2}, \\
D_{j k 2} & =\left(p_{2}-k_{j}-k_{k}\right)^{2}, \\
D_{j 12} & =\left(p_{1}+p_{2}-k_{j}\right)^{2}, \\
D_{k 12} & =\left(p_{1}+p_{2}-k_{k}\right)^{2}, \\
D_{j} & =k_{j}^{2},
\end{aligned}
$$




$$
\begin{aligned}
D_{k} & =k_{k}^{2}, \\
D_{j k 12} & =\left(p_{1}+p_{2}-k_{j}-k_{k}\right)^{2}-q^{2}, \\
D_{j k 123} & =\left(p_{3}+p_{1}+p_{2}-k_{j}-k_{k}\right)^{2},
\end{aligned}
$$

where $p_{3}=x_{2} p_{2}-x_{1} p_{1}$. The cut propagators are $D_{j}, D_{k}, D_{j k 12}, D_{j k 123}$. Only integrals in which all cut propagators appear in the denominator are non-vanishing. Besides the four cut propagators, the phase space integrals of the antenna functions contain at most four further propagators. Any scalar product involving the integration momenta can be written as a linear combination of an appropriately chosen set of seven linear independent propagators (including the four cut propagators). Using this, we are able to express all integrands appearing in the phase space integration of the antenna functions in terms of a linearly independent set of seven of the above propagators. For each of these sets, we derived relations between different integrals based on integration-by-parts identities [105, 106], which are solved by using the Laporta algorithm, using the implementation in [107]. As a result, all phase space integrals can be expressed in terms of 20 independent phase space master integrals. Of these, 10 were already computed in [84].

The set of master integrals which we denote by $I_{i}\left(x_{1}, x_{2}, \epsilon\right)$ are functions of $x_{1}, x_{2}$ and $\epsilon$. We begin by factoring out the leading behaviour of the master integrals $I_{i}\left(x_{1}, x_{2}, \epsilon\right)$ in the limits $x_{1} \rightarrow 1$ and $x_{2} \rightarrow 1$, keeping the exact $\epsilon$-dependence:

$$
I_{i}\left(x_{1}, x_{2}, \epsilon\right)=\left(1-x_{1}\right)^{m_{1}-2 \epsilon}\left(1-x_{2}\right)^{m_{2}-2 \epsilon} \mathcal{F}_{i}\left(x_{1}, x_{2}, \epsilon\right) .
$$

The integers $m_{1}, m_{2}$ are characteristic to each master integral. The functions $\mathcal{F}_{i}\left(x_{1}, x_{2}, \epsilon\right)$ are regular at $x_{1}=1$, at $x_{2}=1$, and at $x_{1}=x_{2}=1$ and can be calculated as Laurent series with, at most, second order poles in $\epsilon$.

The integrated antennae given by $\mathcal{X}\left(x_{1}, x_{2}, \epsilon\right)$ are linear combinations of these master integrals $I_{i}\left(x_{1}, x_{2}, \epsilon\right)$, with coefficients containing poles in $\epsilon$, as well as in $\left(1-x_{1}\right)$ and $\left(1-x_{2}\right)$. After the masters have been inserted into the integrated antennae, those take the form

$$
\mathcal{X}\left(x_{1}, x_{2}, \epsilon\right)=\left(1-x_{1}\right)^{-1-2 \epsilon}\left(1-x_{2}\right)^{-1-2 \epsilon} \mathcal{R}\left(x_{1}, x_{2}, \epsilon\right),
$$

where $\mathcal{R}\left(x_{1}, x_{2}, \epsilon\right)$ is regular at the boundaries $x_{1}=1, x_{2}=1$, and at $x_{1}=x_{2}=1$. The $\epsilon$-expansion of the singular factors $\left(1-x_{i}\right)^{-1-2 \epsilon}$ is done in the form of distributions:

$$
\left(1-x_{i}\right)^{-1-2 \epsilon}=-\frac{1}{2 \epsilon} \delta\left(1-x_{i}\right)+\sum_{n} \frac{(-2 \epsilon)^{n}}{n !} \mathcal{D}_{n}\left(x_{i}\right),
$$

with

$$
\mathcal{D}_{n}\left(x_{i}\right)=\left(\frac{\ln ^{n}\left(1-x_{i}\right)}{1-x_{i}}\right)_{+} .
$$

To evaluate the integrated antennae, we decompose the phase space into four regions depending on the values of $x_{1}$ and $x_{2}$. Those regions are given by:

- $x_{1} \neq 1, x_{2} \neq 1$, which we refer to as the hard region

- $x_{1}=1, x_{2} \neq 1$, and $x_{1} \neq 1, x_{2}=1$, referred to as collinear regions

- $x_{1}=1, x_{2}=1$, which we denote the soft region . 
In the hard region $\left(x_{1} \neq 1, x_{2} \neq 1\right)$, harmonic polylogarithms of weight two appear in the $\mathcal{O}\left(\epsilon^{0}\right)$ term of $\mathcal{R}$. Therefore, the $\epsilon$-expansion of the master integrals in the hard region is needed at least up to the order at which terms of transcendentality two appear.

In the collinear regions $\left(x_{1}=1\right.$ or $\left.x_{2}=1\right)$, since the expansion in distributions in eq. (4.4) generates additional $1 / \epsilon$ factors, the function $\mathcal{R}$ is required up to $\mathcal{O}(\epsilon)$, where harmonic polylogarithms of weight 3 appear. The masters evaluated in the collinear region therefore need to be expanded at least up to this order.

Finally, in the soft region $\left(x_{1}=x_{2}=1\right)$, since the expansion of the distributions in eq. (4.4) generates additional $1 / \epsilon^{2}$ coefficients, the function $\mathcal{R}$ is required up to $\mathcal{O}\left(\epsilon^{2}\right)$ where transcendental constants of weight 4 appear. In this region, we evaluated all master integrals by direct integration. These integrations closely resemble the integrals appearing in the two-loop soft function, and we used the same techniques as were applied in [108], expanding the resulting hypergeometric functions in $\epsilon$ using HypExp [109, 110].

The master integrals in both collinear regions and in the hard region were determined from their differential equations in $x_{1}$ and $x_{2}$, using the results from the soft region as boundary condition. The results for all ten new master integrals can be expressed in terms of harmonic polylogarithms [111] and a few specific combinations of logarithms and polylogarithms. They are listed in the appendix.

Insertion of the master integrals and Laurent expansion of the overall factors according to eq. (4.4) yields the integrated antenna functions $\mathcal{X}\left(x_{1}, x_{2}, \epsilon\right)$.

\section{Results}

The initial-initial antenna functions are listed in tables 2-4. The full results for their integrated forms are too lengthy to be quoted here, a separate FORM file containing those is attached with the arXiv submission of this paper. In this section, we give at most two terms of their Laurent expansion around $\epsilon=0$ below. From each antenna function, we have omitted a common factor $\left(q^{2}\right)^{-2 \epsilon}$. This factor differs from the convention used in [84], where a common factor $\left(s_{12}\right)^{-2 \epsilon}$ had been taken out. It is however in line with the final-final antenna functions derived in [46] and the initial-final antenna functions derived in [82], which are all normalised to $\left(q^{2}\right)^{-2 \epsilon}$.

The leading pole structure of those antenna functions containing a singularity from two soft gluons or from a soft quark-antiquark pair can be predicted from infrared factorisation, and is universal between the final-final, initial-final and initial-initial configurations (in the former two cases, a single antenna function sometimes contains multiple soft gluon limits, which are then additive). For two colour-unconnected (abelian) soft gluons, we expect a leading pole of $1 / \epsilon^{4}$, for two colour-connected (non-abelian) soft gluons a leading pole of $3 / 4 / \epsilon^{4}$ and for a soft quark-antiquark pair a soft pole of $-1 / 12 / \epsilon^{3}$. A similar pattern can be seen in the integrated one-loop antenna functions [46, 82, 83], where the difference between abelian and non-abelian gluonic contributions can be explained by the soft-gluon current at the one-loop order $[16,17]$. The one-loop soft-gluon current contribution cancels between the double real radiation antennae and the one-loop antennae [46-49]. 


\subsection{Quark-antiquark antenna functions}

The leading poles of the integrated initial-initial antenna functions arising from crossings of the quark-antiquark antennae are as follows:

$$
\begin{aligned}
& \mathcal{A}_{12} \equiv \mathcal{A}_{4}^{0}(\widehat{q}, g, g, \widehat{\bar{q}}) \\
& =\frac{1}{\epsilon^{4}} \frac{3}{4} \delta\left(1-x_{1}\right) \delta\left(1-x_{2}\right) \\
& +\frac{1}{\epsilon^{3}}\left[\frac{11}{24} \delta\left(1-x_{1}\right) \delta\left(1-x_{2}\right)+\frac{3}{4} \delta\left(1-x_{2}\right)\left(1+x_{1}-2 \mathcal{D}_{0}\left(x_{1}\right)\right)\right. \\
& \left.+\frac{3}{4} \delta\left(1-x_{1}\right)\left(1+x_{2}-2 \mathcal{D}_{0}\left(x_{2}\right)\right)\right]+\mathcal{O}\left(\epsilon^{-2}\right), \\
& \mathcal{A}_{13} \equiv \mathcal{A}_{4}^{0}(\widehat{q}, \widehat{g}, g, \bar{q}) \\
& =\frac{1}{\epsilon^{3}} \frac{1}{4}\left(1-2 x_{2}+2 x_{2}^{2}\right) \delta\left(1-x_{1}\right)+\mathcal{O}\left(\epsilon^{-2}\right), \\
& \mathcal{A}_{14} \equiv \mathcal{A}_{4}^{0}(\widehat{q}, g, \widehat{g}, \bar{q}) \\
& =\frac{1}{\epsilon^{3}} \frac{1}{2}\left(1-2 x_{2}+2 x_{2}^{2}\right) \delta\left(1-x_{1}\right)+\mathcal{O}\left(\epsilon^{-2}\right), \\
& \mathcal{A}_{34} \equiv \mathcal{A}_{4}^{0}(q, \widehat{g}, \widehat{g}, \bar{q}) \\
& =\frac{1}{\epsilon^{2}} \frac{1}{4}\left(1-2 x_{1}+2 x_{1}^{2}\right)\left(1-2 x_{2}+2 x_{2}^{2}\right)+\mathcal{O}\left(\epsilon^{-1}\right), \\
& \tilde{\mathcal{A}}_{12} \equiv \tilde{\mathcal{A}}_{4}^{0}(\widehat{q}, g, g, \widehat{\bar{q}}) \\
& =\frac{1}{\epsilon^{4}} \delta\left(1-x_{1}\right) \delta\left(1-x_{2}\right) \\
& +\frac{1}{\epsilon^{3}}\left[\delta\left(1-x_{2}\right)\left(1+x_{1}-2 \mathcal{D}_{0}\left(x_{1}\right)\right)+\delta\left(1-x_{1}\right)\left(1+x_{2}-2 \mathcal{D}_{0}\left(x_{2}\right)\right)\right] \\
& +\mathcal{O}\left(\epsilon^{-2}\right) \text {, } \\
& \tilde{\mathcal{A}}_{13} \equiv \tilde{\mathcal{A}}_{4}^{0}(\widehat{q}, \widehat{g}, g, \bar{q}) \\
& =\frac{1}{\epsilon^{3}} \frac{1}{2}\left(1-2 x_{2}+2 x_{2}^{2}\right) \delta\left(1-x_{1}\right)+\mathcal{O}\left(\epsilon^{-2}\right), \\
& \tilde{\mathcal{A}}_{34} \equiv \tilde{\mathcal{A}}_{4}^{0}(q, \widehat{g}, \widehat{g}, \bar{q}) \\
& =\frac{1}{\epsilon^{2}} \frac{1}{2}\left(1-2 x_{1}+2 x_{1}^{2}\right)\left(1-2 x_{2}+2 x_{2}^{2}\right)+\mathcal{O}\left(\epsilon^{-1}\right), \\
& \mathcal{B}_{12} \equiv \mathcal{B}_{4}^{0}\left(\widehat{q}, q^{\prime}, \bar{q}^{\prime}, \widehat{\bar{q}}\right) \\
& =\frac{1}{\epsilon^{3}}\left[-\frac{1}{12} \delta\left(1-x_{1}\right) \delta\left(1-x_{2}\right)\right] \\
& +\frac{1}{\epsilon^{2}}\left[-\frac{5}{36} \delta\left(1-x_{1}\right) \delta\left(1-x_{2}\right)-\frac{1}{12} \delta\left(1-x_{2}\right)\left(1+x_{1}-2 \mathcal{D}_{0}\left(x_{1}\right)\right)\right. \\
& \left.-\frac{1}{12} \delta\left(1-x_{1}\right)\left(1+x_{2}-2 \mathcal{D}_{0}\left(x_{2}\right)\right)\right]+\mathcal{O}\left(\epsilon^{-1}\right), \\
& \mathcal{B}_{13} \equiv \mathcal{B}_{4}^{0}\left(\widehat{q}, \widehat{q^{\prime}}, \bar{q}^{\prime}, \bar{q}\right) \\
& =\frac{1}{\epsilon^{2}} \delta\left(1-x_{1}\right)\left(\frac{1}{4}\left(x_{2}+1\right) H\left(0 ; x_{2}\right)+\frac{\left(1-x_{2}\right)\left(4 x_{2}^{2}+7 x_{2}+4\right)}{24 x_{2}}\right) \\
& +\mathcal{O}\left(\epsilon^{-1}\right) \text {, }
\end{aligned}
$$




$$
\begin{aligned}
\mathcal{B}_{34} \equiv & \mathcal{B}_{4}^{0}\left(q, \widehat{q^{\prime}}, \widehat{\bar{q}^{\prime}}, \widehat{\bar{q}}\right) \\
= & \mathcal{O}\left(\epsilon^{0}\right) \\
\mathcal{C}_{12} \equiv & \mathcal{C}_{4}^{0}(\widehat{q}, \bar{q}, q, \widehat{\bar{q}}) \\
= & \frac{1}{\epsilon}\left[\frac { \delta ( 1 - x _ { 1 } ) } { 4 8 ( 1 - x _ { 2 } ) } \left(\left(1+x_{2}^{2}\right)\left[6 H\left(0,0 ; x_{2}\right)+6 H\left(1,0 ; x_{2}\right)+\pi^{2}\right]\right.\right. \\
& \left.\left.\quad+3\left(5-2 x_{2}^{2}\right) H\left(0 ; x_{2}\right)+3\left(1-x_{2}\right)\left(8-7 x_{2}\right)\right)\right] \\
& +\mathcal{O}\left(\epsilon^{0}\right) \\
\mathcal{C}_{13} \equiv & \mathcal{C}_{4}^{0}(\widehat{q}, \bar{q}, \widehat{q}, \bar{q}) \\
= & \frac{1}{\epsilon}\left[\frac { \delta ( 1 - x _ { 1 } ) } { 2 4 ( 1 + x _ { 2 } ) } \left(\left(1+x_{2}^{2}\right)\left[12 H\left(-1,0 ; x_{2}\right)-6 H\left(0,0 ; x_{2}\right)+\pi^{2}\right]\right.\right. \\
& \left.\left.\quad-12\left(1-x_{2}^{2}\right)-6\left(1+x_{2}\right)^{2} H\left(0 ; x_{2}\right)\right)\right]+\mathcal{O}\left(\epsilon^{0}\right), \\
\mathcal{C}_{23} \equiv & \mathcal{C}_{4}^{0}(q, \widehat{\bar{q}}, \widehat{q}, \bar{q}) \\
= & \mathcal{O}\left(\epsilon^{0}\right), \\
\mathcal{C}_{24} \equiv & \mathcal{C}_{4}^{0}(q, \bar{q}, \widehat{q}, \widehat{\bar{q}}) \\
= & \mathcal{O}\left(\epsilon^{0}\right) .
\end{aligned}
$$

We observe that $\mathcal{B}_{34}, \mathcal{C}_{23}$ and $\mathcal{C}_{34}$ are finite, as expected. Moreover, the leading pole structure of $\mathcal{A}_{12}$ (non-abelian), $\tilde{\mathcal{A}}_{12}$ (abelian) and $\mathcal{B}_{12}$ is as predicted from the double soft factorisation. $\mathcal{B}_{12}, \mathcal{B}_{13}$ and $\mathcal{B}_{34}$ were computed previously in [84].

\subsection{Quark-gluon antenna functions}

The leading poles of the integrated initial-initial antenna functions obtained from crossing quark-gluon antennae read:

$$
\begin{aligned}
\mathcal{D}_{12} \equiv & \mathcal{D}_{4}^{0}(\widehat{q}, \widehat{g}, g, g) \\
= & \frac{1}{\epsilon^{4}} \frac{3}{4} \delta\left(1-x_{1}\right) \delta\left(1-x_{2}\right) \\
& +\frac{1}{\epsilon^{3}}\left[\frac{11}{24} \delta\left(1-x_{1}\right) \delta\left(1-x_{2}\right)+\frac{3}{4} \delta\left(1-x_{2}\right)\left(1+x_{1}-2 \mathcal{D}_{0}\left(x_{1}\right)\right)\right. \\
& \left.\quad \quad+\frac{3}{2} \delta\left(1-x_{1}\right)\left(x_{2}^{2}-x_{2}-\frac{1}{x_{2}}+2-\mathcal{D}_{0}\left(x_{2}\right)\right)\right]+\mathcal{O}\left(\epsilon^{-2}\right), \\
\mathcal{D}_{13} \equiv & \mathcal{D}_{4}^{0}(\widehat{q}, g, \widehat{g}, g) \\
= & \frac{1}{\epsilon^{4}} \delta\left(1-x_{1}\right) \delta\left(1-x_{2}\right) \\
& +\frac{1}{\epsilon^{3}}\left[\delta\left(1-x_{2}\right)\left(1+x_{1}-2 \mathcal{D}_{0}\left(x_{1}\right)\right)\right. \\
& \left.\quad+2 \delta\left(1-x_{1}\right)\left(x_{2}^{2}-x_{2}-\frac{1}{x_{2}}+2-\mathcal{D}_{0}\left(x_{2}\right)\right)\right]+\mathcal{O}\left(\epsilon^{-2}\right),
\end{aligned}
$$




$$
\begin{aligned}
\mathcal{D}_{23} \equiv & \mathcal{D}_{4}^{0}(q, \widehat{g}, \widehat{g}, g) \\
= & \frac{1}{\epsilon^{3}}\left[\frac{1}{4}\left(1-2 x_{2}+2 x_{2}^{2}\right) \delta\left(1-x_{1}\right)+\frac{1}{2}\left(1-2 x_{1}+2 x_{1}^{2}\right) \delta\left(1-x_{2}\right)\right] \\
& +\mathcal{O}\left(\epsilon^{-2}\right), \\
\mathcal{D}_{24} \equiv & \mathcal{D}_{4}^{0}(q, \widehat{g}, g, \widehat{g}) \\
= & \frac{1}{\epsilon^{3}}\left[\frac{1}{2}\left(1-2 x_{2}+2 x_{2}^{2}\right) \delta\left(1-x_{1}\right)+\frac{1}{2}\left(1-2 x_{1}+2 x_{1}^{2}\right) \delta\left(1-x_{2}\right)\right] \\
& +\mathcal{O}\left(\epsilon^{-2}\right), \\
\mathcal{E}_{12} \equiv & \mathcal{E}_{4}^{0}\left(\widehat{q}, \widehat{q^{\prime}}, \bar{q}^{\prime}, g\right) \\
= & \frac{1}{\epsilon^{3}} \frac{\left(2-2 x_{2}+x_{2}^{2}\right) \delta\left(1-x_{1}\right)}{2 x_{2}}+\mathcal{O}\left(\epsilon^{-2}\right), \\
\mathcal{E}_{14} \equiv & \mathcal{E}_{4}^{0}\left(\widehat{q}, q^{\prime}, \bar{q}^{\prime}, \widehat{g}\right) \\
= & \frac{1}{\epsilon^{3}}\left[-\frac{1}{12} \delta\left(1-x_{1}\right) \delta\left(1-x_{2}\right)\right] \\
& +\frac{1}{\epsilon^{2}}\left[-\frac{5}{36} \delta\left(1-x_{1}\right) \delta\left(1-x_{2}\right)-\frac{1}{12} \delta\left(1-x_{2}\right)\left(1+x_{1}-2 \mathcal{D}_{0}\left(x_{1}\right)\right)\right. \\
& \quad+\delta\left(1-x_{1}\right)\left(\frac{6 x_{2} H\left(0 ; x_{2}\right)\left(1+x_{2}\right)-8 x_{2}^{3}+x_{2}^{2}-5 x_{2}+8}{24 x_{2}}\right. \\
& \left.\left.\quad \frac{1}{6} \mathcal{D}_{0}\left(x_{2}\right)\right)\right]+\mathcal{O}\left(\epsilon^{-1}\right),
\end{aligned}
$$

$\mathcal{E}_{23} \equiv \mathcal{E}_{4}^{0}\left(q, \widehat{q^{\prime}}, \widehat{\bar{q}^{\prime}}, g\right)$

$$
=\frac{1}{\epsilon^{2}} \frac{x_{1} x_{2}\left(1+x_{1} x_{2}\right)^{2}\left(x_{1}^{2}+x_{2}^{2}-2\right)}{\left(x_{1}+x_{2}\right)^{4}}+\mathcal{O}\left(\epsilon^{-1}\right),
$$

$\mathcal{E}_{24} \equiv \mathcal{E}_{4}^{0}\left(q, \widehat{q^{\prime}}, \bar{q}^{\prime}, \widehat{g}\right)$

$$
\begin{aligned}
=\frac{1}{\epsilon^{2}} & {\left[\delta\left(1-x_{2}\right)\left(\frac{1}{4}\left(1+x_{1}\right) H\left(0 ; x_{1}\right)+\frac{\left(1-x_{1}\right)\left(4 x_{1}^{2}+7 x_{1}+4\right)}{24 x_{1}}\right)\right.} \\
& \left.+\frac{\left(2-2 x_{1}+x_{1}^{2}\right)\left(1-2 x_{2}+2 x_{2}^{2}\right)}{4 x_{1}}\right]+\mathcal{O}\left(\epsilon^{-1}\right),
\end{aligned}
$$

$\tilde{\mathcal{E}}_{12} \equiv \tilde{\mathcal{E}}_{4}^{0}\left(\widehat{q}, \widehat{q^{\prime}}, \bar{q}^{\prime}, g\right)$

$$
\begin{aligned}
= & \frac{1}{\epsilon^{3}} \frac{\left(2-2 x_{2}+x_{2}^{2}\right) \delta\left(1-x_{1}\right)}{4 x_{2}}+\mathcal{O}\left(\epsilon^{-2}\right), \\
\tilde{\mathcal{E}}_{14} \equiv & \tilde{\mathcal{E}}_{4}^{0}\left(\widehat{q}, q^{\prime}, \bar{q}^{\prime}, \widehat{g}\right) \\
= & \frac{1}{\epsilon^{2}} \delta\left(1-x_{1}\right)\left(\frac{1}{2}\left(1+x_{2}\right) H\left(0 ; x_{2}\right)+\frac{\left(1-x_{2}\right)\left(4 x_{2}^{2}+7 x_{2}+4\right)}{12 x_{2}}\right) \\
& +\mathcal{O}\left(\epsilon^{-1}\right), \\
\tilde{\mathcal{E}}_{23} \equiv & \tilde{\mathcal{E}}_{4}^{0}\left(q, \widehat{q^{\prime}}, \widehat{\bar{q}^{\prime}}, g\right) \\
= & \frac{1}{\epsilon^{2}} \frac{x_{1} x_{2}\left(1+x_{1} x_{2}\right)^{2}\left(x_{1}^{2}+x_{2}^{2}-2\right)}{\left(x_{1}+x_{2}\right)^{4}}+\mathcal{O}\left(\epsilon^{-1}\right),
\end{aligned}
$$




$$
\begin{aligned}
\tilde{\mathcal{E}}_{24} \equiv \tilde{\mathcal{E}}_{4}^{0}\left(q, \widehat{q}^{\prime}, \bar{q}^{\prime}, \widehat{g}\right) & \\
=\frac{1}{\epsilon}[ & \frac{\left(2 x_{1}^{8}+x_{2} x_{1}^{7}-x_{1}^{6}+2 x_{2}^{2} x_{1}^{4}-x_{2} x_{1}^{3}-8 x_{2}^{2} x_{1}^{2}+2 x_{2} x_{1}+8 x_{2}^{2}\right)}{x_{1}^{4}} \\
& \cdot\left(G\left(-x_{2} ; x_{1}\right)-G\left(-1 ; x_{1}\right)\right)+\left(2 x_{1}^{2}+x_{2} x_{1}-1\right) x_{1}^{2} H\left(0 ; x_{2}\right) \\
& +\frac{\left(1-x_{2}\right)}{6\left(x_{1}+x_{2}\right)^{3} x_{1}^{3}}\left(12 x_{1}^{9}+36 x_{2} x_{1}^{8}-6 x_{1}^{8}+41 x_{2}^{2} x_{1}^{7}-19 x_{2} x_{1}^{7}\right. \\
& -x_{1}^{7}+19 x_{2}^{3} x_{1}^{6}-29 x_{2}^{2} x_{1}^{6}-5 x_{2} x_{1}^{6}+4 x_{2}^{4} x_{1}^{5}-20 x_{2}^{3} x_{1}^{5}-26 x_{2}^{2} x_{1}^{5} \\
& +15 x_{2} x_{1}^{5}-6 x_{2}^{4} x_{1}^{4}-30 x_{2}^{3} x_{1}^{4}+73 x_{2}^{2} x_{1}^{4}+10 x_{2} x_{1}^{4}-2 x_{1}^{4}-12 x_{2}^{4} x_{1}^{3} \\
& +86 x_{2}^{3} x_{1}^{3}+50 x_{2}^{2} x_{1}^{3}-34 x_{2} x_{1}^{3}+32 x_{2}^{4} x_{1}^{2}+62 x_{2}^{3} x_{1}^{2}-118 x_{2}^{2} x_{1}^{2} \\
& \left.\left.+24 x_{2}^{4} x_{1}-132 x_{2}^{3} x_{1}-48 x_{2}^{4}\right)\right] \\
+\mathcal{O} & \left(\epsilon^{0}\right) .
\end{aligned}
$$

Again, the leading pole structure of $\mathcal{D}_{12}$ (non-abelian), $\mathcal{D}_{13}$ (abelian) and $\mathcal{E}_{14}$ is as expected. $\tilde{\mathcal{E}}_{12}, \tilde{\mathcal{E}}_{14}, \tilde{\mathcal{E}}_{23}, \tilde{\mathcal{E}}_{24}$ were computed already in [84].

\subsection{Gluon-gluon antenna functions}

Finally, the leading poles of the integrated initial-initial gluon-gluon antenna functions are:

$$
\begin{aligned}
& \mathcal{F}_{12} \equiv \mathcal{F}_{4}^{0}(\widehat{g}, \widehat{g}, g, g) \\
&= \frac{1}{\epsilon^{4}} \frac{3}{4} \delta\left(1-x_{1}\right) \delta\left(1-x_{2}\right) \\
&+\frac{1}{\epsilon^{3}}\left[\frac{11}{24} \delta\left(1-x_{1}\right) \delta\left(1-x_{2}\right)+\frac{3}{2} \delta\left(1-x_{2}\right)\left(x_{1}^{2}-x_{1}-\frac{1}{x_{1}}+2-\mathcal{D}_{0}\left(x_{1}\right)\right)\right. \\
&\left.\quad+\frac{3}{2} \delta\left(1-x_{1}\right)\left(x_{2}^{2}-x_{2}-\frac{1}{x_{2}}+2-\mathcal{D}_{0}\left(x_{2}\right)\right)\right]+\mathcal{O}\left(\epsilon^{-2}\right) \\
& \mathcal{F}_{13} \equiv \mathcal{F}_{4}^{0}(\widehat{g}, g, \widehat{g}, g) \\
&= \frac{1}{\epsilon^{4}} \delta\left(1-x_{1}\right) \delta\left(1-x_{2}\right) \\
&+\frac{1}{\epsilon^{3}}\left[2 \delta\left(1-x_{1}\right)\left(x_{2}^{2}-x_{2}-\frac{1}{x_{2}}+2-\mathcal{D}_{0}\left(x_{2}\right)\right)\right. \\
&\left.\quad+2 \delta\left(1-x_{2}\right)\left(x_{1}^{2}-x_{1}-\frac{1}{x_{1}}+2-\mathcal{D}_{0}\left(x_{1}\right)\right)\right]+\mathcal{O}\left(\epsilon^{-2}\right) \\
& \mathcal{G}_{12} \equiv \mathcal{G}_{4}^{0}(\widehat{g}, q, \bar{q}, \widehat{g}) \\
&= \frac{1}{\epsilon^{3}}\left[-\frac{1}{12} \delta\left(1-x_{1}\right) \delta\left(1-x_{2}\right)\right] \\
&+\frac{1}{\epsilon^{2}}\left[\frac{-5}{36} \delta\left(1-x_{1}\right) \delta\left(1-x_{2}\right)\right. \\
& \quad+\delta\left(1-x_{1}\right)\left(\frac{6 x_{2} H\left(0 ; x_{2}\right)\left(1+x_{2}\right)-8 x_{2}^{3}+x_{2}^{2}-5 x_{2}+8}{24 x_{2}}\right. \\
&\left.\quad+\frac{1}{6} \mathcal{D}_{0}\left(x_{2}\right)\right)
\end{aligned}
$$




$$
\begin{aligned}
& +\delta\left(1-x_{2}\right)\left(\frac{6 x_{1} H\left(0 ; x_{1}\right)\left(1+x_{1}\right)-8 x_{1}^{3}+x_{1}^{2}-5 x_{1}+8}{24 x_{1}}\right. \\
& \left.\left.+\frac{1}{6} \mathcal{D}_{0}\left(x_{1}\right)\right)\right]+\mathcal{O}\left(\epsilon^{-1}\right) \\
& \mathcal{G}_{13} \equiv \mathcal{G}_{4}^{0}(\widehat{g}, \widehat{q}, \bar{q}, g) \\
& =\frac{1}{\epsilon^{3}} \frac{\left(2-2 x_{2}+x_{2}^{2}\right) \delta\left(1-x_{1}\right)}{2 x_{2}}+\mathcal{O}\left(\epsilon^{-2}\right), \\
& \mathcal{G}_{14} \equiv \mathcal{G}_{4}^{0}(\widehat{g}, q, \widehat{\bar{q}}, g) \\
& =\frac{1}{\epsilon^{3}} \frac{\left(2-2 x_{2}+x_{2}^{2}\right) \delta\left(1-x_{1}\right)}{2 x_{2}}+\mathcal{O}\left(\epsilon^{-2}\right), \\
& \mathcal{G}_{34} \equiv \mathcal{G}_{4}^{0}(g, \widehat{q}, \widehat{\bar{q}}, g) \\
& =\frac{1}{\epsilon^{2}} \frac{2\left(1+x_{1} x_{2}\right)\left(x_{2}^{2} x_{1}^{4}+x_{2}^{4} x_{1}^{2}-4 x_{2}^{2} x_{1}^{2}+x_{1}^{2}+x_{2}^{2}\right)}{\left(x_{1}+x_{2}\right)^{4}}+\mathcal{O}\left(\epsilon^{-1}\right), \\
& \tilde{\mathcal{G}}_{12} \equiv \tilde{\mathcal{G}}_{4}^{0}(\widehat{g}, q, \bar{q}, \widehat{g}) \\
& =\frac{1}{\epsilon^{2}}\left[\delta\left(1-x_{2}\right)\left(\frac{1}{2}\left(1+x_{1}\right) H\left(0 ; x_{1}\right)+\frac{\left(1-x_{1}\right)\left(4 x_{1}^{2}+7 x_{1}+4\right)}{12 x_{1}}\right)\right. \\
& \left.+\delta\left(1-x_{1}\right)\left(\frac{1}{2}\left(1+x_{2}\right) H\left(0 ; x_{2}\right)+\frac{\left(1-x_{2}\right)\left(4 x_{2}^{2}+7 x_{2}+4\right)}{12 x_{2}}\right)\right] \\
& +\mathcal{O}\left(\epsilon^{-1}\right), \\
& \tilde{\mathcal{G}}_{13} \equiv \tilde{\mathcal{G}}_{4}^{0}(\widehat{g}, \widehat{q}, \bar{q}, g) \\
& =\frac{1}{\epsilon^{3}} \frac{\left(2-2 x_{2}+x_{2}^{2}\right) \delta\left(1-x_{1}\right)}{4 x_{2}}+\mathcal{O}\left(\epsilon^{-2}\right), \\
& \tilde{\mathcal{G}}_{34} \equiv \tilde{\mathcal{G}}_{4}^{0}(g, \widehat{q}, \widehat{\bar{q}}, g) \\
& =\frac{1}{\epsilon^{2}} \frac{2\left(1+x_{1} x_{2}\right)\left(x_{2}^{2} x_{1}^{4}+x_{2}^{4} x_{1}^{2}-4 x_{2}^{2} x_{1}^{2}+x_{1}^{2}+x_{2}^{2}\right)}{\left(x_{1}+x_{2}\right)^{4}}+\mathcal{O}\left(\epsilon^{-1}\right), \\
& \mathcal{H}_{12} \equiv \mathcal{H}_{4}^{0}\left(\widehat{q}, \widehat{\bar{q}}, q^{\prime}, \bar{q}^{\prime}\right) \\
& =\frac{1}{\epsilon}\left[-\frac{\left(1+x_{1} x_{2}\right)\left(x_{2}^{2} x_{1}^{4}+x_{2}^{4} x_{1}^{2}-4 x_{2}^{2} x_{1}^{2}+x_{1}^{2}+x_{2}^{2}\right)}{3\left(x_{1}+x_{2}\right)^{4}}\right]+\mathcal{O}\left(\epsilon^{0}\right), \\
& \mathcal{H}_{13} \equiv \mathcal{H}_{4}^{0}\left(\widehat{q}, \bar{q}, \widehat{q^{\prime}}, \bar{q}^{\prime}\right) \\
& =\frac{1}{\epsilon^{2}} \frac{\left(2-2 x_{1}+x_{1}^{2}\right)\left(2-2 x_{2}+x_{2}^{2}\right)}{4 x_{1} x_{2}}+\mathcal{O}\left(\epsilon^{-1}\right) .
\end{aligned}
$$

The leading pole structure of $\mathcal{F}_{12}$ (non-abelian), $\mathcal{F}_{13}$ (abelian) and $\mathcal{G}_{12}$ is as predicted from the double soft factorisation. $\mathcal{H}_{12}$ and $\mathcal{H}_{13}$ were computed already in [84].

\section{Conclusions}

In this paper, we have completed the integration of double real radiation antenna functions in the kinematical situation where both radiator partons are in the initial state. A subset of our results, restricted to antenna functions containing a secondary fermion pair, was 
obtained earlier in [84]. With the results presented in this paper, combined with the finalfinal [46], initial-final [82] and virtual one-loop initial-initial antennae [83], all antenna functions required for the calculation of jet cross sections at hadron colliders are now available in unintegrated and integrated form. The implementation of the corresponding antenna subtraction terms into a numerical parton-level event generator program is thus readily feasible.

Using the general method completed in this paper, NNLO QCD calculations for exclusive jet observables at hadron colliders are now in reach. The principal requirement for these calculations is the availability of the relevant two-loop matrix elements. Potential applications, where two-loop matrix elements have already been derived, are: two-jet production [112-120], vector-boson-plus-jet production [121-123] and Higgs-boson-plus-jet production [124]. Important steps have already been taken in the calculation of two-jet production at NNLO, where the unintegrated subtraction terms for purely gluonic processes have already been derived and tested for the double real radiation at tree-level [80] and the single real radiation at one-loop [81], and in the incorporation of processes with quarks into this calculation [125]. Finally, we notice that the integrated initial-initial antenna functions $\mathcal{F}_{12}$ and $\mathcal{F}_{13}$ derived here appear in the integrated subtraction terms of the all-gluon contribution to two-jet production. They contribute to the cancellation of all infrared poles when combined with all other integrated subtraction terms and with the virtual two-loop matrix elements [126]. This pole cancellation has been verified [126] and can be regarded as a strong check on the correctness of our results presented here for these integrated antennae $\mathcal{F}_{12}$ and $\mathcal{F}_{13}$.

\section{Acknowledgments}

We would like to thank Nigel Glover and Joao Pires for many stimulating discussions. Part of this work was done while AG and TG attended the "Physics with first data from the LHC" program at the Kavli Institute for Theoretical Physics (KITP) at UC Santa Barbara. This research is supported in part by the Swiss National Science Foundation (SNF) under contracts PP00P2-139192 and 200020-138206, and by the Research Executive Agency (REA) of the European Union under the Grant Agreement number PITN-GA2010-264564 (LHCPhenoNet). The work of MR was supported under European Research Council Advanced Investigator Grant ERC-AdG-228301.

\section{A Master integrals}

The phase space integrals appearing in the integration of the initial-initial four-parton antenna functions can be reduced to a set of 20 independent master integrals, as described in section 4. Of these integrals, the following 10 were derived previously in [84]:

$$
\begin{aligned}
I_{1} & =\int\left[\mathrm{d} k_{j}\right]\left[\mathrm{d} k_{k}\right] \delta\left(C_{1}\right) \delta\left(C_{2}\right)\left(k_{k} \cdot p_{1}\right), \\
I_{2} & =\int\left[\mathrm{d} k_{j}\right]\left[\mathrm{d} k_{k}\right] \delta\left(C_{1}\right) \delta\left(C_{2}\right),
\end{aligned}
$$




$$
\begin{aligned}
I_{3} & =\int\left[\mathrm{d} k_{j}\right]\left[\mathrm{d} k_{k}\right] \delta\left(C_{1}\right) \delta\left(C_{2}\right) \frac{-\left(k_{j} \cdot p_{1}\right)\left(k_{j} \cdot p_{3}\right)}{D_{k 12}}, \\
I_{4} & =\int\left[\mathrm{d} k_{j}\right]\left[\mathrm{d} k_{k}\right] \delta\left(C_{1}\right) \delta\left(C_{2}\right) \frac{\left(k_{j} \cdot p_{1}\right)}{D_{k 12}}, \\
I_{5} & =\int\left[\mathrm{d} k_{j}\right]\left[\mathrm{d} k_{k}\right] \delta\left(C_{1}\right) \delta\left(C_{2}\right) \frac{-\left(k_{j} \cdot p_{3}\right)}{D_{k 12}}, \\
I_{6} & =\int\left[\mathrm{d} k_{j}\right]\left[\mathrm{d} k_{k}\right] \delta\left(C_{1}\right) \delta\left(C_{2}\right) \frac{1}{D_{k 12}}, \\
I_{7} & =\int\left[\mathrm{d} k_{j}\right]\left[\mathrm{d} k_{k}\right] \delta\left(C_{1}\right) \delta\left(C_{2}\right) \frac{1}{D_{j k 2}} \\
I_{8} & =\int\left[\mathrm{d} k_{j}\right]\left[\mathrm{d} k_{k}\right] \delta\left(C_{1}\right) \delta\left(C_{2}\right) \frac{1}{D_{k 2}}, \\
I_{14} & =\int\left[\mathrm{d} k_{j}\right]\left[\mathrm{d} k_{k}\right] \delta\left(C_{1}\right) \delta\left(C_{2}\right) \frac{1}{D_{j 12} D_{j k 2}}, \\
I_{15} & =\int\left[\mathrm{d} k_{j}\right]\left[\mathrm{d} k_{k}\right] \delta\left(C_{1}\right) \delta\left(C_{2}\right) \frac{1}{D_{j 1} D_{k 2}} .
\end{aligned}
$$

The remaining 10 integrals are:

$$
\begin{aligned}
I_{13} & =\int\left[\mathrm{d} k_{j}\right]\left[\mathrm{d} k_{k}\right] \delta\left(C_{1}\right) \delta\left(C_{2}\right) \frac{1}{D_{k 2} D_{j 12}}, \\
I_{16} & =\int\left[\mathrm{d} k_{j}\right]\left[\mathrm{d} k_{k}\right] \delta\left(C_{1}\right) \delta\left(C_{2}\right) \frac{1}{D_{j k} D_{j 1} D_{j 12}}, \\
I_{17} & =\int\left[\mathrm{d} k_{j}\right]\left[\mathrm{d} k_{k}\right] \delta\left(C_{1}\right) \delta\left(C_{2}\right) \frac{1}{D_{j 1} D_{k 2} D_{j k 2}}, \\
I_{22} & =\int\left[\mathrm{d} k_{j}\right]\left[\mathrm{d} k_{k}\right] \delta\left(C_{1}\right) \delta\left(C_{2}\right) \frac{1}{D_{j 1} D_{k 2} D_{j 12}}, \\
I_{23} & =\int\left[\mathrm{d} k_{j}\right]\left[\mathrm{d} k_{k}\right] \delta\left(C_{1}\right) \delta\left(C_{2}\right) \frac{1}{D_{k 2} D_{j k 2} D_{j 12}}, \\
I_{24} & =\int\left[\mathrm{d} k_{j}\right]\left[\mathrm{d} k_{k}\right] \delta\left(C_{1}\right) \delta\left(C_{2}\right) \frac{1}{D_{j 1} D_{j k 2} D_{j 12}}, \\
I_{25} & =\int\left[\mathrm{d} k_{j}\right]\left[\mathrm{d} k_{k}\right] \delta\left(C_{1}\right) \delta\left(C_{2}\right) \frac{1}{D_{j 2} D_{k 2} D_{k 12}}, \\
I_{27} & =\int\left[\mathrm{d} k_{j}\right]\left[\mathrm{d} k_{k}\right] \delta\left(C_{1}\right) \delta\left(C_{2}\right) \frac{1}{D_{j 1} D_{j 2} D_{j k 2}}, \\
I_{28} & =\int\left[\mathrm{d} k_{j}\right]\left[\mathrm{d} k_{k}\right] \delta\left(C_{1}\right) \delta\left(C_{2}\right) \frac{1}{D_{j 1} D_{j 2} D_{k 2}}, \\
I_{29} & =\int\left[\mathrm{d} k_{j}\right]\left[\mathrm{d} k_{k}\right] \delta\left(C_{1}\right) \delta\left(C_{2}\right) \frac{1}{D_{j 2} D_{j k} D_{k 1}}
\end{aligned}
$$

Note that, the numeration of the integrals is not consecutive, since masters related by exchange of $x_{1}$ and $x_{2}$ are listed only for one crossing in both sets.

In the new set, the integrals $I_{13}, I_{22}, I_{23}$ and $I_{25}$ appear always in particular combinations, which can be made explicit by introducing new master integrals $M_{22}, M_{23}$ and 
$M_{25}$ by:

$$
\begin{aligned}
& I_{22}=M_{22}+\frac{1}{2 x_{1} x_{2} s_{12}} I_{13}, \\
& I_{23}=M_{23}+\frac{1-2 x_{1}^{2}-2 x_{1} x_{2}}{2 x_{2}\left(1-x_{1}^{2}\right)\left(x_{1}+x_{2}\right) s_{12}} I_{13}, \\
& I_{25}=M_{25}+\frac{x_{1}\left(x_{1}+2 x_{2}\right)}{2 x_{2}\left(1-x_{1}^{2}\right) s_{12}} I_{13} .
\end{aligned}
$$

After expressing the integrated antenna functions in this new basis it turns out that the remaining coefficients of $I_{13}$ are all proportional to $\epsilon$, such that $I_{13}$ is required only to lower order in $\epsilon$ than the other master integrals.

\section{A.1 Hard region}

In the hard region, the new master integrals are:

$$
\begin{aligned}
I_{13}= & \left(s_{12}\right)^{-2-2 \epsilon} S_{\Gamma} \frac{\left(1-x_{1}\right)^{-2 \epsilon}\left(1-x_{2}\right)^{-2 \epsilon}}{x_{1}} \frac{1}{\epsilon} g_{13}\left(x_{1}, x_{2}\right)+\mathcal{O}\left(\epsilon^{0}\right), \\
I_{16}= & \left(s_{12}\right)^{-3-2 \epsilon} S_{\Gamma} \frac{\left(1-x_{1}\right)^{-2 \epsilon}\left(1-x_{2}\right)^{-2 \epsilon}}{x_{1}\left(1-x_{2}^{2}\right)}[ \\
& -\frac{1}{\epsilon^{2}}+\frac{1}{\epsilon}\left(-3 G\left(-x_{2} ; x_{1}\right)+G\left(-1 ; x_{1}\right)+2 H\left(-1 ; x_{2}\right)-3 H\left(0 ; x_{2}\right)\right) \\
& +\frac{17 \pi^{2}}{12}-2 G\left(1 / x_{2} ; x_{1}\right) H\left(0 ; x_{2}\right)-2 G\left(1 / x_{2}, 0 ; x_{1}\right)-6 G\left(-x_{2},-x_{2} ; x_{1}\right) \\
& +3 G\left(-x_{2} ; x_{1}\right) H\left(-1 ; x_{2}\right)-6 G\left(-x_{2} ; x_{1}\right) H\left(0 ; x_{2}\right)+3 G\left(-x_{2},-1 ; x_{1}\right) \\
& +3 G\left(-1,-x_{2} ; x_{1}\right)+G\left(-1 ; x_{1}\right) \log 2-2 G\left(-1 ; x_{1}\right) H\left(-1 ; x_{2}\right)+3 G\left(-1 ; x_{1}\right) H\left(0 ; x_{2}\right) \\
& -2 G\left(-1,-1 ; x_{1}\right)+2 G\left(0 ; x_{1}\right) H\left(0 ; x_{2}\right)+4 G\left(0,0 ; x_{1}\right)-3 G\left(1,-x_{2} ; x_{1}\right) \\
& -G\left(1 ; x_{1}\right) \log 2+3 G\left(1 ; x_{1}\right) H\left(-1 ; x_{2}\right)-3 G\left(1 ; x_{1}\right) H\left(0 ; x_{2}\right)+G\left(1,-1 ; x_{1}\right) \\
& -2 G\left(1,0 ; x_{1}\right)-4 H\left(-1,-1 ; x_{2}\right)+6 H\left(-1,0 ; x_{2}\right)+3 H\left(0,-1 ; x_{2}\right) \\
& \left.-4 H\left(0,0 ; x_{2}\right)\right]+\mathcal{O}(\epsilon), \\
& \left(s_{12}\right)^{-3-2 \epsilon} S_{\Gamma} \frac{\left(1-x_{1}\right)^{-2 \epsilon}\left(1-x_{2}\right)^{-2 \epsilon}}{x_{1} x_{2}^{2}\left(1-x_{1}\right)}\left[-\frac{1}{2 \epsilon^{2}}\right. \\
& +\frac{1}{\epsilon}\left(\log 2-G\left(-1 ; x_{1}\right)+G\left(0 ; x_{1}\right)+H\left(0 ; x_{2}\right)\right) \\
& -2 \log { }^{2} 2-\frac{\pi^{2}}{2}+G\left(1 / x_{2} ; x_{1}\right) H\left(-1 ; x_{2}\right)-G\left(1 / x_{2},-1 ; x_{1}\right)+G\left(1 / x_{2}, 0 ; x_{1}\right) \\
& -3 G\left(-1,-x_{2} ; x_{1}\right)+2 G\left(-1 ; x_{1}\right) \log 2-G\left(-1 ; x_{1}\right) H\left(-1 ; x_{2}\right)-G\left(-1 ; x_{1}\right) H\left(0 ; x_{2}\right) \\
& +2 G\left(-1,-1 ; x_{1}\right)+G\left(-1,0 ; x_{1}\right)-2 G\left(0 ; x_{1}\right) \log 2-2 G\left(0 ; x_{1}\right) H\left(0 ; x_{2}\right) \\
& +2 G\left(0,-1 ; x_{1}\right)-2 G\left(0,0 ; x_{1}\right)-2 H\left(0 ; x_{2}\right) \log 2-2 H\left(0,0 ; x_{2}\right)-2 H\left(1 ; x_{2}\right) \log 2 \\
& \left.+2 H\left(1,-1 ; x_{2}\right)-4 H\left(1,0 ; x_{2}\right)\right]
\end{aligned}
$$




$$
\begin{aligned}
& +\left(s_{12}\right)^{-3-2 \epsilon} S_{\Gamma} \frac{\left(1-x_{1}\right)^{-2 \epsilon}\left(1-x_{2}\right)^{-2 \epsilon}}{x_{1} x_{2}^{2}\left(1+x_{1}\right)}\left[\frac{1}{\epsilon}\left(-\log 2+H\left(-1 ; x_{2}\right)\right)\right. \\
& +2 \log ^{2} 2+\frac{\pi^{2}}{6}+G\left(1 / x_{2} ; x_{1}\right) H\left(-1 ; x_{2}\right)-G\left(1 / x_{2},-1 ; x_{1}\right)+G\left(1 / x_{2}, 0 ; x_{1}\right) \\
& +2 G\left(-1 ; x_{1}\right) \log 2-2 G\left(-1 ; x_{1}\right) H\left(-1 ; x_{2}\right)+2 G\left(0 ; x_{1}\right) \log 2-2 G\left(0 ; x_{1}\right) H\left(-1 ; x_{2}\right) \\
& -3 G\left(1,-x_{2} ; x_{1}\right)-4 G\left(1 ; x_{1}\right) \log 2+3 G\left(1 ; x_{1}\right) H\left(-1 ; x_{2}\right)-3 G\left(1 ; x_{1}\right) H\left(0 ; x_{2}\right) \\
& +4 G\left(1,-1 ; x_{1}\right)-G\left(1,0 ; x_{1}\right)-2 H\left(-1,-1 ; x_{2}\right)+2 H\left(-1,0 ; x_{2}\right)+2 H\left(0 ; x_{2}\right) \log 2 \\
& \left.-2 H\left(0,-1 ; x_{2}\right)+2 H\left(1 ; x_{2}\right) \log 2-2 H\left(1,-1 ; x_{2}\right)\right]+\mathcal{O}(\epsilon), \\
& M_{22}=\left(s_{12}\right)^{-3-2 \epsilon} S_{\Gamma} \frac{\left(1-x_{1}\right)^{-2 \epsilon}\left(1-x_{2}\right)^{-2 \epsilon}}{x_{1}^{2} x_{2}}[ \\
& \frac{1}{2 \epsilon^{2}}+\frac{1}{\epsilon}\left(\frac{1}{2} G\left(-x_{2} ; x_{1}\right)-\frac{1}{2} G\left(-1 ; x_{1}\right)-G\left(0 ; x_{1}\right)+\frac{1}{2} H\left(0 ; x_{2}\right)\right) \\
& -\frac{7 \pi^{2}}{24}-\frac{1}{2} G\left(-x_{2} ; x_{1}\right) H\left(-1 ; x_{2}\right)+G\left(-x_{2} ; x_{1}\right) H\left(0 ; x_{2}\right)+\frac{1}{2} G\left(-x_{2},-1 ; x_{1}\right) \\
& -G\left(-x_{2}, 0 ; x_{1}\right)-\frac{3}{2} G\left(-1,-x_{2} ; x_{1}\right)-\frac{1}{2} G\left(-1 ; x_{1}\right) \log 2+G\left(-1 ; x_{1}\right) H\left(-1 ; x_{2}\right) \\
& -\frac{3}{2} G\left(-1 ; x_{1}\right) H\left(0 ; x_{2}\right)+G\left(-1,-1 ; x_{1}\right)-G\left(0,-x_{2} ; x_{1}\right)-G\left(0 ; x_{1}\right) H\left(0 ; x_{2}\right) \\
& +G\left(0,-1 ; x_{1}\right)+2 G\left(0,0 ; x_{1}\right)-\frac{1}{2} G\left(1,-x_{2} ; x_{1}\right)+\frac{1}{2} G\left(1 ; x_{1}\right) \log 2 \\
& +\frac{1}{2} G\left(1 ; x_{1}\right) H\left(-1 ; x_{2}\right)-\frac{1}{2} G\left(1 ; x_{1}\right) H\left(0 ; x_{2}\right)-\frac{1}{2} G\left(1,-1 ; x_{1}\right)-G\left(1,0 ; x_{1}\right) \\
& -2 H\left(-1 ; x_{2}\right) \log 2+H\left(-1,-1 ; x_{2}\right)-H\left(-1,0 ; x_{2}\right)-\frac{3}{2} H\left(0,-1 ; x_{2}\right)+H\left(0,0 ; x_{2}\right) \\
& \left.-2 H\left(1 ; x_{2}\right) \log 2+2 H\left(1,-1 ; x_{2}\right)-3 H\left(1,0 ; x_{2}\right)-g_{22}\left(x_{1}, x_{2}\right)\right]+\mathcal{O}(\epsilon), \\
& M_{23}=\left(s_{12}\right)^{-3-2 \epsilon} S_{\Gamma} \frac{\left(1-x_{1}\right)^{-2 \epsilon}\left(1-x_{2}\right)^{-2 \epsilon}}{x_{1} x_{2}\left(1-x_{1}^{2}\right)\left(x_{1}+x_{2}\right)}[ \\
& \frac{1}{\epsilon}\left(\frac{1}{2} G\left(-x_{2} ; x_{1}\right)-\frac{1}{2} G\left(-1 ; x_{1}\right)+\frac{3}{2} H\left(0 ; x_{2}\right)\right) \\
& -\frac{29 \pi^{2}}{24}-G\left(-x_{2},-x_{2} ; x_{1}\right)-\frac{1}{2} G\left(-x_{2} ; x_{1}\right) H\left(-1 ; x_{2}\right)-2 G\left(-x_{2} ; x_{1}\right) H\left(0 ; x_{2}\right) \\
& +\frac{3}{2} G\left(-x_{2},-1 ; x_{1}\right)-G\left(-x_{2}, 0 ; x_{1}\right)-\frac{5}{2} G\left(-1,-x_{2} ; x_{1}\right)+\frac{1}{2} G\left(-1 ; x_{1}\right) \log 2 \\
& -\frac{5}{2} G\left(-1 ; x_{1}\right) H\left(0 ; x_{2}\right)+2 G\left(-1,-1 ; x_{1}\right)+G\left(-1,0 ; x_{1}\right)-G\left(0,-x_{2} ; x_{1}\right) \\
& -3 G\left(0 ; x_{1}\right) H\left(0 ; x_{2}\right)+G\left(0,-1 ; x_{1}\right)-\frac{3}{2} G\left(1,-x_{2} ; x_{1}\right)-\frac{1}{2} G\left(1 ; x_{1}\right) \log 2 \\
& +\frac{1}{2} G\left(1 ; x_{1}\right) H\left(-1 ; x_{2}\right)+\frac{1}{2} G\left(1 ; x_{1}\right) H\left(0 ; x_{2}\right)+\frac{3}{2} G\left(1,-1 ; x_{1}\right)-2 H\left(-1 ; x_{2}\right) \log 2 \\
& -H\left(-1 ; x_{2}\right) H\left(1 ; x_{1}\right)+H\left(-1,-1 ; x_{2}\right)-3 H\left(-1,0 ; x_{2}\right)-2 H\left(0 ; x_{2}\right) \log 2
\end{aligned}
$$




$$
\begin{aligned}
& +2 H\left(0 ; x_{2}\right) H\left(1 ; x_{1}\right)+\frac{5}{2} H\left(0,-1 ; x_{2}\right)-6 H\left(0,0 ; x_{2}\right)+H\left(1 ; x_{1}\right) \log 2 \\
& -3 H\left(1 ; x_{2}\right) \log 2+3 H\left(1,-1 ; x_{2}\right)-6 H\left(1,0 ; x_{2}\right)+4 g_{23 b}\left(x_{2}\right)-2 g_{22}\left(x_{1}, x_{2}\right) \\
& \left.+g_{23}\left(x_{1}, x_{2}\right)+g_{25}\left(x_{1}, x_{2}\right)\right]+\mathcal{O}(\epsilon) \text {, } \\
& I_{24}=\left(s_{12}\right)^{-3-2 \epsilon} S_{\Gamma} \frac{\left(1-x_{1}\right)^{-2 \epsilon}\left(1-x_{2}\right)^{-2 \epsilon}}{x_{1} x_{2}\left(x_{1}-x_{2}\right)}\left[\frac{1}{\epsilon}\left(-G\left(-1 ; x_{1}\right)+H\left(-1 ; x_{2}\right)\right)\right. \\
& +\frac{\pi^{2}}{6}+2 G\left(1 / x_{2} ; x_{1}\right) H\left(-1 ; x_{2}\right)-2 G\left(1 / x_{2},-1 ; x_{1}\right)+2 G\left(1 / x_{2}, 0 ; x_{1}\right) \\
& -3 G\left(-1,-x_{2} ; x_{1}\right)+G\left(-1 ; x_{1}\right) \log 2-3 G\left(-1 ; x_{1}\right) H\left(0 ; x_{2}\right)+2 G\left(-1,-1 ; x_{1}\right) \\
& -2 G\left(0 ; x_{1}\right) H\left(-1 ; x_{2}\right)+2 G\left(0,-1 ; x_{1}\right)-3 G\left(1,-x_{2} ; x_{1}\right)-G\left(1 ; x_{1}\right) \log 2 \\
& +3 G\left(1 ; x_{1}\right) H\left(-1 ; x_{2}\right)-3 G\left(1 ; x_{1}\right) H\left(0 ; x_{2}\right)+G\left(1,-1 ; x_{1}\right)-2 G\left(1,0 ; x_{1}\right) \\
& -3 H\left(-1 ; x_{2}\right) \log 2-2 H\left(-1,-1 ; x_{2}\right)+3 H\left(-1,0 ; x_{2}\right)-3 H\left(1 ; x_{2}\right) \log 2 \\
& \left.+3 H\left(1,-1 ; x_{2}\right)-3 H\left(1,0 ; x_{2}\right)+2 g_{24}\left(x_{1} ; x_{2}\right)\right]+\mathcal{O}(\epsilon), \\
& M_{25}=\left(s_{12}\right)^{-3-2 \epsilon} S_{\Gamma} \frac{\left(1-x_{1}\right)^{-2 \epsilon}\left(1-x_{2}\right)^{-2 \epsilon}}{x_{2}\left(1-x_{1}^{2}\right)}[ \\
& \frac{1}{\epsilon}\left(\frac{1}{2} G\left(-x_{2} ; x_{1}\right)-\frac{1}{2} G\left(-1 ; x_{1}\right)+\frac{3}{2} H\left(0 ; x_{2}\right)\right) \\
& -\frac{23 \pi^{2}}{24}-\frac{1}{2} G\left(-x_{2} ; x_{1}\right) H\left(-1 ; x_{2}\right)+G\left(-x_{2} ; x_{1}\right) H\left(0 ; x_{2}\right)+\frac{1}{2} G\left(-x_{2},-1 ; x_{1}\right) \\
& -G\left(-x_{2}, 0 ; x_{1}\right)-\frac{5}{2} G\left(-1,-x_{2} ; x_{1}\right)+\frac{1}{2} G\left(-1 ; x_{1}\right) \log 2-\frac{5}{2} G\left(-1 ; x_{1}\right) H\left(0 ; x_{2}\right) \\
& +2 G\left(-1,-1 ; x_{1}\right)+G\left(-1,0 ; x_{1}\right)+G\left(0,-x_{2} ; x_{1}\right)-G\left(0 ; x_{1}\right) H\left(0 ; x_{2}\right)-G\left(0,-1 ; x_{1}\right) \\
& -\frac{3}{2} G\left(1,-x_{2} ; x_{1}\right)-\frac{1}{2} G\left(1 ; x_{1}\right) \log 2+\frac{1}{2} G\left(1 ; x_{1}\right) H\left(-1 ; x_{2}\right)+\frac{1}{2} G\left(1 ; x_{1}\right) H\left(0 ; x_{2}\right) \\
& +\frac{3}{2} G\left(1,-1 ; x_{1}\right)-H\left(-1 ; x_{2}\right) \log 2-H\left(-1 ; x_{2}\right) H\left(1 ; x_{1}\right)-H\left(-1,0 ; x_{2}\right) \\
& -2 H\left(0 ; x_{2}\right) \log 2+2 H\left(0 ; x_{2}\right) H\left(1 ; x_{1}\right)+\frac{5}{2} H\left(0,-1 ; x_{2}\right)-5 H\left(0,0 ; x_{2}\right) \\
& +H\left(1 ; x_{1}\right) \log 2-2 H\left(1 ; x_{2}\right) \log 2+2 H\left(1,-1 ; x_{2}\right)-5 H\left(1,0 ; x_{2}\right) \\
& \left.+4 g_{23 b}\left(x_{2}\right)-g_{22}\left(x_{1}, x_{2}\right)+g_{23}\left(x_{1}, x_{2}\right)+g_{25}\left(x_{1}, x_{2}\right)\right]+\mathcal{O}(\epsilon), \\
& I_{27}=\left(s_{12}\right)^{-3-2 \epsilon} S_{\Gamma} \frac{\left(1-x_{1}\right)^{-2 \epsilon}\left(1-x_{2}\right)^{-2 \epsilon}}{x_{2}\left(1-x_{1}^{2}\right)}[ \\
& -\frac{1}{\epsilon^{2}}+\frac{1}{\epsilon}\left(-2 G\left(-x_{2} ; x_{1}\right)+2 G\left(-1 ; x_{1}\right)-2 H\left(0 ; x_{2}\right)\right) \\
& +\frac{3 \pi^{2}}{2}-2 G\left(1 / x_{2} ; x_{1}\right) H\left(-1 ; x_{2}\right)+2 G\left(1 / x_{2},-1 ; x_{1}\right)-2 G\left(1 / x_{2}, 0 ; x_{1}\right) \\
& +4 G\left(-1,-x_{2} ; x_{1}\right)+4 G\left(-1 ; x_{1}\right) H\left(0 ; x_{2}\right)-4 G\left(-1,-1 ; x_{1}\right)+2 G\left(0 ; x_{1}\right) H\left(-1 ; x_{2}\right)
\end{aligned}
$$




$$
\begin{aligned}
& -2 G\left(0,-1 ; x_{1}\right)+2 G\left(0,0 ; x_{1}\right)+2 H\left(-1 ; x_{2}\right) \log 2+2 H\left(0,0 ; x_{2}\right) \\
& \left.+2 H\left(1 ; x_{2}\right) \log 2-2 H\left(1,-1 ; x_{2}\right)+6 H\left(1,0 ; x_{2}\right)\right]+\mathcal{O}(\epsilon), \\
& I_{28}=\left(s_{12}\right)^{-3-2 \epsilon} S_{\Gamma} \frac{\left(1-x_{1}\right)^{-2 \epsilon}\left(1-x_{2}\right)^{-2 \epsilon}}{x_{1} x_{2}\left(1-x_{1}^{2}\right)}[ \\
& -\frac{1}{2 \epsilon^{2}}+\frac{1}{\epsilon}\left(2 \log 2-G\left(-1 ; x_{1}\right)+G\left(0 ; x_{1}\right)-H\left(-1 ; x_{2}\right)+H\left(0 ; x_{2}\right)\right) \\
& -4 \log ^{2} 2-\frac{2 \pi^{2}}{3}-3 G\left(-1,-x_{2} ; x_{1}\right)+G\left(-1 ; x_{1}\right) H\left(-1 ; x_{2}\right)-G\left(-1 ; x_{1}\right) H\left(0 ; x_{2}\right) \\
& +2 G\left(-1,-1 ; x_{1}\right)+G\left(-1,0 ; x_{1}\right)-4 G\left(0 ; x_{1}\right) \log 2+2 G\left(0 ; x_{1}\right) H\left(-1 ; x_{2}\right) \\
& -2 G\left(0 ; x_{1}\right) H\left(0 ; x_{2}\right)+2 G\left(0,-1 ; x_{1}\right)-2 G\left(0,0 ; x_{1}\right)+3 G\left(1,-x_{2} ; x_{1}\right) \\
& +4 G\left(1 ; x_{1}\right) \log 2-3 G\left(1 ; x_{1}\right) H\left(-1 ; x_{2}\right)+3 G\left(1 ; x_{1}\right) H\left(0 ; x_{2}\right)-4 G\left(1,-1 ; x_{1}\right) \\
& +G\left(1,0 ; x_{1}\right)+2 H\left(-1,-1 ; x_{2}\right)-2 H\left(-1,0 ; x_{2}\right)-4 H\left(0 ; x_{2}\right) \log 2+2 H\left(0,-1 ; x_{2}\right) \\
& \left.-2 H\left(0,0 ; x_{2}\right)-4 H\left(1 ; x_{2}\right) \log 2+4 H\left(1,-1 ; x_{2}\right)-4 H\left(1,0 ; x_{2}\right)\right] \\
& +\left(s_{12}\right)^{-3-2 \epsilon} S_{\Gamma} \frac{\left(1-x_{1}\right)^{-2 \epsilon}\left(1-x_{2}\right)^{-2 \epsilon}}{x_{2}\left(1-x_{1}^{2}\right)}[ \\
& -\frac{3}{2 \epsilon^{2}}+\frac{1}{\epsilon}\left(-2 G\left(-x_{2} ; x_{1}\right)+G\left(-1 ; x_{1}\right)+G\left(0 ; x_{1}\right)+H\left(-1 ; x_{2}\right)-H\left(0 ; x_{2}\right)\right) \\
& +\pi^{2}+G\left(-1,-x_{2} ; x_{1}\right)+4 G\left(-1 ; x_{1}\right) \log 2-3 G\left(-1 ; x_{1}\right) H\left(-1 ; x_{2}\right) \\
& +3 G\left(-1 ; x_{1}\right) H\left(0 ; x_{2}\right)-2 G\left(-1,-1 ; x_{1}\right)+G\left(-1,0 ; x_{1}\right)+2 G\left(0,-x_{2} ; x_{1}\right) \\
& -2 G\left(0,-1 ; x_{1}\right)-3 G\left(1,-x_{2} ; x_{1}\right)-4 G\left(1 ; x_{1}\right) \log 2+3 G\left(1 ; x_{1}\right) H\left(-1 ; x_{2}\right) \\
& -3 G\left(1 ; x_{1}\right) H\left(0 ; x_{2}\right)+4 G\left(1,-1 ; x_{1}\right)-G\left(1,0 ; x_{1}\right)+2 H\left(-1 ; x_{2}\right) \log 2 \\
& -2 H\left(-1,-1 ; x_{2}\right)+2 H\left(-1,0 ; x_{2}\right)+2 H\left(1 ; x_{2}\right) \log 2-2 H\left(1,-1 ; x_{2}\right) \\
& \left.+2 H\left(1,0 ; x_{2}\right)\right]+\mathcal{O}(\epsilon) \\
& I_{29}=\left(s_{12}\right)^{-3-2 \epsilon} S_{\Gamma} \frac{\left(1-x_{1}\right)^{-2 \epsilon}\left(1-x_{2}\right)^{-2 \epsilon}}{x_{1} x_{2}\left(1+x_{1}\right)\left(1+x_{2}\right)}[ \\
& \frac{1}{2 \epsilon^{2}}+\frac{1}{\epsilon}\left(-2 \log 2+G\left(-1 ; x_{1}\right)-G\left(0 ; x_{1}\right)+H\left(-1 ; x_{2}\right)-H\left(0 ; x_{2}\right)\right) \\
& +4 \log ^{2} 2+\frac{2 \pi^{2}}{3}+3 G\left(-1,-x_{2} ; x_{1}\right)-G\left(-1 ; x_{1}\right) H\left(-1 ; x_{2}\right)+G\left(-1 ; x_{1}\right) H\left(0 ; x_{2}\right) \\
& -2 G\left(-1,-1 ; x_{1}\right)-G\left(-1,0 ; x_{1}\right)+4 G\left(0 ; x_{1}\right) \log 2-2 G\left(0 ; x_{1}\right) H\left(-1 ; x_{2}\right) \\
& +2 G\left(0 ; x_{1}\right) H\left(0 ; x_{2}\right)-2 G\left(0,-1 ; x_{1}\right)+2 G\left(0,0 ; x_{1}\right)-3 G\left(1,-x_{2} ; x_{1}\right) \\
& -4 G\left(1 ; x_{1}\right) \log 2+3 G\left(1 ; x_{1}\right) H\left(-1 ; x_{2}\right)-3 G\left(1 ; x_{1}\right) H\left(0 ; x_{2}\right)+4 G\left(1,-1 ; x_{1}\right) \\
& -G\left(1,0 ; x_{1}\right)-2 H\left(-1,-1 ; x_{2}\right)+2 H\left(-1,0 ; x_{2}\right)+4 H\left(0 ; x_{2}\right) \log 2-2 H\left(0,-1 ; x_{2}\right) \\
& \left.+2 H\left(0,0 ; x_{2}\right)+4 H\left(1 ; x_{2}\right) \log 2-4 H\left(1,-1 ; x_{2}\right)+4 H\left(1,0 ; x_{2}\right)\right]
\end{aligned}
$$




$$
\begin{aligned}
& +\left(s_{12}\right)^{-3-2 \epsilon} S_{\Gamma} \frac{\left(x_{1}+x_{2}\right)\left(1-x_{1}\right)^{-2 \epsilon}\left(1-x_{2}\right)^{-2 \epsilon}}{x_{1} x_{2}\left(1-x_{1}^{2}\right)\left(1-x_{2}^{2}\right)}[ \\
& \frac{3}{\epsilon^{2}}+\frac{2}{\epsilon}\left(-\log 2+3 G\left(-x_{2} ; x_{1}\right)-G\left(-1 ; x_{1}\right)-2 G\left(0 ; x_{1}\right)-H\left(-1 ; x_{2}\right)+H\left(0 ; x_{2}\right)\right) \\
& +4 \log ^{2} 2-\frac{4 \pi^{2}}{3}+\log 2\left(-4 G\left(-1 ; x_{1}\right)+4 G\left(0 ; x_{1}\right)-4 H\left(-1 ; x_{2}\right)+4 H\left(0 ; x_{2}\right)\right) \\
& +12 G\left(-x_{2},-x_{2} ; x_{1}\right)-6 G\left(-x_{2} ; x_{1}\right) H\left(-1 ; x_{2}\right)+6 G\left(-x_{2} ; x_{1}\right) H\left(0 ; x_{2}\right) \\
& -6 G\left(-x_{2},-1 ; x_{1}\right)-6 G\left(-x_{2}, 0 ; x_{1}\right)-6 G\left(-1,-x_{2} ; x_{1}\right)+6 G\left(-1 ; x_{1}\right) H\left(-1 ; x_{2}\right) \\
& -6 G\left(-1 ; x_{1}\right) H\left(0 ; x_{2}\right)+4 G\left(-1,-1 ; x_{1}\right)+2 G\left(-1,0 ; x_{1}\right)-6 G\left(0,-x_{2} ; x_{1}\right) \\
& +2 G\left(0,-1 ; x_{1}\right)+4 G\left(0,0 ; x_{1}\right)+4 H\left(-1,-1 ; x_{2}\right)-4 H\left(-1,0 ; x_{2}\right)-4 H\left(0,-1 ; x_{2}\right) \\
& \left.+4 H\left(0,0 ; x_{2}\right)\right]+\mathcal{O}(\epsilon) .
\end{aligned}
$$

In the above equations, we introduced the functions:

$$
\begin{aligned}
z\left(x_{1}, x_{2}\right)= & \sqrt{1+4 x_{1} x_{2}+4 x_{2}^{2}}, \\
g_{13}\left(x_{1}, x_{2}\right)= & \frac{1}{z\left(x_{1}, x_{2}\right)}\left[\log \left(\frac{z\left(x_{1}, x_{2}\right)+1-2 x_{2}}{z\left(x_{1}, x_{2}\right)-1+2 x_{2}}\right)+\log \left(\frac{z\left(x_{1}, x_{2}\right)+1}{z\left(x_{1}, x_{2}\right)-1}\right)\right] \\
g_{22}\left(x_{1}, x_{2}\right)= & \int_{1}^{x_{1}} \mathrm{~d} y_{1} \frac{g_{13}\left(y_{1}, x_{2}\right)}{y_{1}+x_{2}} \\
= & \frac{\pi^{2}}{12}+\frac{3}{2} \log ^{2} x_{2}+\log 2 \log \left(\frac{1-x_{2}}{1+x_{2}}\right)-2 \log x_{2} \log \left(1+x_{2}\right)+\frac{1}{2} \log \left(1+x_{2}\right)^{2} \\
& +\log \left(\frac{1-x_{2}}{x_{2}}\right) \log (z-1)-\frac{1}{2} \log ^{2}\left(\frac{z-1}{z+1}\right)+\log \left(x_{2}\right) \log \left(1-2 x_{2}+z\right) \\
& -\log \left(1-x_{2}\right) \log \left(z-1+2 x_{2}\right)+\log \left(\frac{z+1}{2}\right) \log \left(\frac{z-1+2 x_{2}}{z+1-2 x_{2}}\right) \\
& +\operatorname{Li} 2\left(-\frac{1}{x_{2}}\right)-\operatorname{Li} 2\left(-\frac{4 x_{2}}{2-2 x_{2}}\right)+\operatorname{Li} 2\left(-\frac{2 x_{2}}{2-2 x_{2}}\right) \\
& -\operatorname{Li} 2\left(\frac{1-z}{2-2 x_{2}}\right)+\operatorname{Li} 2\left(\frac{1-z}{2 x_{2}}\right)+\operatorname{Li} 2\left(\frac{1-2 x_{2}-z}{2-2 x_{2}}\right) \\
& -\operatorname{Li}_{2}\left(-\frac{1-2 x_{2}+z}{2 x_{2}}\right), \\
& \int_{1}^{x_{1}} \mathrm{~d} y_{1}\left(g_{13}\left(y_{1}, x_{2}\right)-g_{13}\left(1, x_{2}\right)\right) \frac{-1-2 x_{2}}{1-y_{1}} \\
= & \log 2 \log \left(1+\frac{1}{2 x_{2}}\right)+\log \left(2+\frac{1}{x_{2}}\right) \log \left(2\left(1+x_{2}\right)\right) \\
& +\log \left(2 x_{2}\right)\left(\log \left(1+x_{2}\right)-\log \left(1+2 x_{2}\right)\right)-\log \left(4 x_{2}\right) \log \left(1+2 x_{2}\right) \\
& +\log \left(-1+2 x_{2}+z\right) \log \left(\frac{1+2 x_{2}+z}{2}\right) \\
& +\log (1+z)\left(\log 2+\log \left(x_{2}\right)-\log \left(1+2 x_{2}+z\right)\right) \\
& +\log \left(1-2 x_{2}+z\right)\left(\log 4+\log \left(x_{2}\right)-\log \left(1+2 x_{2}+z\right)\right) \\
g_{23}\left(x_{1}, x_{2}\right) & \text { A. } 18 \\
&
\end{aligned}
$$




$$
\begin{aligned}
& +\log \left(\frac{1+x_{2}}{2(z-1) x_{2}^{2}}\right) \log \left(\frac{2+4 x_{2}}{1+2 x_{2}+z}\right) \\
& +\mathrm{Li}_{2}\left(-\frac{1}{2 x_{2}}\right)-\mathrm{Li}_{2}\left(-2 x_{2}\right)+\mathrm{Li}_{2}\left(-\frac{1+x_{2}}{x_{2}}\right)-\mathrm{Li}_{2}\left(-\frac{x_{2}}{1+x_{2}}\right) \\
& +\mathrm{Li}_{2}\left(\frac{1-z}{2+2 x_{2}}\right)+\operatorname{Li}_{2}\left(\frac{1-2 x_{2}-z}{2}\right)+\operatorname{Li}_{2}\left(\frac{1+2 x_{2}-z}{4 x_{2}}\right) \\
& +\mathrm{Li}_{2}\left(\frac{1+2 x_{2}-z}{2 x_{2}}\right)-\mathrm{Li}_{2}\left(\frac{1+2 x_{2}-z}{2+2 x_{2}}\right)-\mathrm{Li}_{2}\left(\frac{1+2 x_{2}-z}{2}\right) \\
& -\mathrm{Li}_{2}\left(-\frac{1+z}{2 x_{2}}\right)-\mathrm{Li}_{2}\left(-\frac{1-2 x_{2}+z}{4 x_{2}}\right), \\
& g_{23 b}\left(x_{2}\right)=\int_{1}^{x_{2}} \mathrm{~d} y_{2} \frac{1}{1+2 y_{2}}\left(H\left(-1 ; y_{2}\right)-\log 2-2 H\left(0 ; y_{2}\right)\right) \\
& =\frac{\pi^{2}}{6}+\log \left(2 x_{2}\right) \log \left(1+2 x_{2}\right)+\frac{1}{2} \operatorname{Li}_{2}\left(-1-2 x_{2}\right)+\operatorname{Li}_{2}\left(-2 x_{2}\right), \\
& g_{23 c}\left(x_{2}\right)=\int_{1}^{x_{2}} \mathrm{~d} y_{2}\left(\frac{1}{y_{2}}-\frac{1}{1+y_{2}}\right) g_{23 b}\left(y_{2}\right) \\
& =-\frac{\pi^{2}}{12} \log 2+\frac{17}{6} \log ^{3} 2+\frac{\pi^{2}}{6} \log 3-2 \log ^{2} 2 \log 3+\frac{1}{6} \log ^{3} 3+\frac{\pi^{2}}{6} \log \left(x_{2}\right) \\
& -\frac{1}{4} \log ^{2} 2 \log \left(x_{2}\right)-\frac{1}{12} \log ^{3}\left(x_{2}\right)+\frac{\pi^{2}}{12} \log \left(1+x_{2}\right)+\frac{7}{4} \log ^{2} 2 \log \left(1+x_{2}\right) \\
& -\frac{1}{2} \log 2 \log \left(x_{2}\right) \log \left(1+x_{2}\right)-\frac{1}{4} \log ^{2}\left(x_{2}\right) \log \left(1+x_{2}\right)+2 \log 2 \log ^{2}\left(1+x_{2}\right) \\
& +\frac{7}{4} \log \left(x_{2}\right) \log ^{2}\left(1+x_{2}\right)-\frac{1}{12} \log ^{3}\left(1+x_{2}\right)+\frac{\pi^{2}}{6} \log \left(1+2 x_{2}\right) \\
& -\frac{1}{2} \log ^{2} 2 \log \left(1+2 x_{2}\right)+\frac{1}{2} \log 2 \log \left(x_{2}\right) \log \left(1+2 x_{2}\right) \\
& +\frac{1}{2} \log ^{2}\left(x_{2}\right) \log \left(1+2 x_{2}\right)-\frac{3}{2} \log 2 \log \left(1+x_{2}\right) \log \left(1+2 x_{2}\right) \\
& -2 \log \left(x_{2}\right) \log \left(1+x_{2}\right) \log \left(1+2 x_{2}\right)-\log ^{2}\left(1+x_{2}\right) \log \left(1+2 x_{2}\right) \\
& +\frac{3}{4} \log 2 \log ^{2}\left(1+2 x_{2}\right)+\log \left(x_{2}\right) \log ^{2}\left(1+2 x_{2}\right)+\frac{7}{4} \log \left(1+x_{2}\right) \log ^{2}\left(1+2 x_{2}\right) \\
& -\log ^{3}\left(1+2 x_{2}\right)+\frac{1}{2} \log 2 \log \left(\frac{1+2 x_{2}}{2+2 x_{2}}\right) \log \left(2+2 x_{2}\right) \\
& +\frac{1}{2} \log \left(1+x_{2}\right) \log \left(\frac{1+2 x_{2}}{2+2 x_{2}}\right) \log \left(2+2 x_{2}\right)+\frac{3}{2} \log \left(x_{2}\right) \operatorname{Li}_{2}\left(\frac{x_{2}}{1+x_{2}}\right) \\
& -\frac{3}{2} \log \left(1+x_{2}\right) \operatorname{Li}_{2}\left(\frac{x_{2}}{1+x_{2}}\right)+\log \left(x_{2}\right) \operatorname{Li}_{2}\left(\frac{x_{2}}{1+2 x_{2}}\right) \\
& -\log \left(1+2 x_{2}\right) \operatorname{Li}_{2}\left(\frac{x_{2}}{1+2 x_{2}}\right)+\frac{1}{2} \log 2 \operatorname{Li}_{2}\left(\frac{2 x_{2}}{1+2 x_{2}}\right) \\
& -\frac{1}{2} \log \left(x_{2}\right) \operatorname{Li}_{2}\left(\frac{2 x_{2}}{1+2 x_{2}}\right)+\log \left(1+x_{2}\right) \operatorname{Li}_{2}\left(\frac{2 x_{2}}{1+2 x_{2}}\right) \\
& -\frac{1}{2} \log \left(x_{2}\right) \operatorname{Li}_{2}\left(\frac{1+x_{2}}{1+2 x_{2}}\right)+\frac{3}{2} \log \left(1+x_{2}\right) \operatorname{Li}_{2}\left(\frac{1+x_{2}}{1+2 x_{2}}\right)
\end{aligned}
$$




$$
\begin{aligned}
& -\log \left(1+2 x_{2}\right) \mathrm{Li}_{2}\left(\frac{1+x_{2}}{1+2 x_{2}}\right)+\frac{1}{2} \log 2 \mathrm{Li}_{2}\left(\frac{1+2 x_{2}}{2+2 x_{2}}\right) \\
& -\frac{1}{2} \log \left(x_{2}\right) \operatorname{Li}_{2}\left(\frac{1+2 x_{2}}{2+2 x_{2}}\right)+\log \left(1+x_{2}\right) \operatorname{Li}_{2}\left(\frac{1+2 x_{2}}{2+2 x_{2}}\right) \\
& -2 \mathrm{Li}_{3}\left(-\frac{1}{2}\right)-\mathrm{Li}_{3}\left(-\frac{1}{3}\right)-\frac{1}{2} \mathrm{Li}_{3}\left(\frac{1}{4}\right)-\mathrm{Li}_{3}\left(\frac{3}{4}\right)+\mathrm{Li}_{3}\left(-1-2 x_{2}\right) \\
& +\frac{3}{2} \mathrm{Li}_{3}\left(-2 x_{2}\right)+\mathrm{Li}_{3}\left(-x_{2}\right)+\mathrm{Li}_{3}\left(\frac{1}{1+x_{2}}\right)-\frac{1}{2} \mathrm{Li}_{3}\left(\frac{x_{2}}{1+x_{2}}\right) \\
& +\frac{1}{2} \mathrm{Li}_{3}\left(-\frac{1+x_{2}}{x_{2}}\right)+\mathrm{Li}_{3}\left(\frac{1}{1+2 x_{2}}\right)-\mathrm{Li}_{3}\left(\frac{x_{2}}{1+2 x_{2}}\right)+\mathrm{Li}_{3}\left(\frac{2 x_{2}}{1+2 x_{2}}\right) \\
& -\mathrm{Li}_{3}\left(\frac{1+x_{2}}{1+2 x_{2}}\right)+\frac{1}{2} \operatorname{Li}_{3}\left(\frac{1}{2+2 x_{2}}\right)+\mathrm{Li}_{3}\left(\frac{1+2 x_{2}}{2+2 x_{2}}\right)+\frac{5}{16} \zeta_{3}, \\
& g_{24}\left(x_{1}, x_{2}\right)=\int_{1}^{x_{1}} \mathrm{~d} y_{1} \frac{\log \left(1+y_{1}\right)-\log \left(1+x_{2}\right)}{y_{1}-x_{2}} \\
& =\frac{\log ^{2} 2}{2}-\frac{1}{2} \log ^{2}\left(1+x_{1}\right)-\log 2 \log \left(1-x_{2}\right)+\log \left(1+x_{1}\right) \log \left(x_{1}-x_{2}\right) \\
& +\log \left(1-x_{2}\right) \log \left(1+x_{2}\right)-\log \left(x_{1}-x_{2}\right) \log \left(1+x_{2}\right)+\operatorname{Li}_{2}\left(\frac{1+x_{2}}{2}\right) \\
& -\mathrm{Li}_{2}\left(\frac{1+x_{2}}{1+x_{1}}\right) \\
& g_{25}\left(x_{1}, x_{2}\right)=\int_{1}^{x_{1}} \mathrm{~d} y_{1} g_{13}\left(y_{1}, x_{2}\right) \frac{1-2 x_{2}}{1+y_{1}} \\
& =-\frac{\pi^{2}}{12}+\frac{\log ^{2} 2}{2}+\log \left(-1+\frac{1}{x_{2}}\right) \log \left(2-2 x_{2}\right)-\log ^{2}\left(2-2 x_{2}\right) \\
& +\log \left(2-2 x_{2}\right) \log \left(x_{2}\right)+\frac{1}{2} \log \left(x_{2}\right) \log \left(4 x_{2}\right)-\log \left(2-2 x_{2}\right) \log (z-1) \\
& +\log \left(2 x_{2}\right) \log (z-1)-\log \left(2 x_{2}\right) \log \left(\frac{1-2 x_{2}+z}{2}\right) \\
& +\log (z-1) \log \left(1-2 x_{2}+z\right)-\frac{1}{2} \log ^{2}\left(1-2 x_{2}+z\right) \\
& +\log \left(2-2 x_{2}\right) \log \left(-1+2 x_{2}+z\right)-\log (z-1) \log \left(-1+2 x_{2}+z\right) \\
& +\log \left(1-2 x_{2}+z\right) \log \left(-1+2 x_{2}+z\right)-\frac{1}{2} \log ^{2}\left(-1+2 x_{2}+z\right) \\
& -\mathrm{Li}_{2}\left(-\frac{1}{x_{2}}\right)-\mathrm{Li}_{2}\left(-\frac{x_{2}}{1-x_{2}}\right)+\mathrm{Li}_{2}\left(-\frac{2 x_{2}}{1-x_{2}}\right)+\mathrm{Li}_{2}\left(-\frac{z-1}{2\left(1-x_{2}\right)}\right) \\
& -\mathrm{Li}_{2}\left(-\frac{z-1}{2 x_{2}}\right)+\mathrm{Li}_{2}\left(-\frac{-2 x_{2}+z+1}{2 x_{2}}\right)-\mathrm{Li}_{2}\left(-\frac{2 x_{2}+z-1}{2\left(1-x_{2}\right)}\right) \text {, }
\end{aligned}
$$

with $z \equiv z\left(x_{1}, x_{2}\right)$.

\section{A.2 Collinear $x_{1}$ region}

In the collinear $x_{1}$ region, we can expand around $x_{2}=1$, and the master integrals read:

$$
I_{13}=\mathcal{O}\left(\left(1-x_{2}\right)\right) \text {, }
$$




$$
\begin{aligned}
I_{16}= & \left(s_{12}\right)^{-3-2 \epsilon} S_{\Gamma} \frac{\left(1-x_{1}\right)^{-2 \epsilon}\left(1-x_{2}\right)^{-1-2 \epsilon}}{2 x_{1}}[ \\
& -\frac{1}{\epsilon^{2}}+\frac{1}{\epsilon}\left(2 \log 2-2 H\left(-1 ; x_{1}\right)\right)+\left(-2 \log ^{2} 2+\frac{7 \pi^{2}}{6}+2 H\left(-1 ; x_{1}\right) \log 2\right. \\
& \left.-2 H\left(-1,-1 ; x_{1}\right)+4 H\left(0,0 ; x_{1}\right)-2 H\left(1 ; x_{1}\right) \log 2+2 H\left(1,-1 ; x_{1}\right)+4 H\left(1,0 ; x_{1}\right)\right) \\
& +\epsilon\left(\frac{33}{4} \zeta_{3}+\frac{4}{3} \log ^{3} 2+\log ^{2} 2\left(-H\left(-1 ; x_{1}\right)+3 H\left(1 ; x_{1}\right)\right)+\log 2\left(2 H\left(-1,-1 ; x_{1}\right)\right.\right. \\
& \left.+4 H\left(-1,0 ; x_{1}\right)-8 H\left(0,0 ; x_{1}\right)-2 H\left(1,-1 ; x_{1}\right)-4 H\left(1,0 ; x_{1}\right)+4 H\left(1,1 ; x_{1}\right)\right) \\
& +\frac{7}{3} \pi^{2}\left(-\log 2-H\left(1 ; x_{1}\right)+H\left(-1 ; x_{1}\right)-H\left(0 ; x_{1}\right)\right)-2 H\left(-1,-1,-1 ; x_{1}\right) \\
& -4 H\left(-1,0,-1 ; x_{1}\right)+8 H\left(-1,0,0 ; x_{1}\right)+8 H\left(-1,1,0 ; x_{1}\right)+4 H\left(0,-1,0 ; x_{1}\right) \\
& +8 H\left(0,0,-1 ; x_{1}\right)-16 H\left(0,0,0 ; x_{1}\right)-12 H\left(0,1,0 ; x_{1}\right)+2 H\left(1,-1,-1 ; x_{1}\right) \\
& +4 H\left(1,-1,0 ; x_{1}\right)+4 H\left(1,0,-1 ; x_{1}\right)-16 H\left(1,0,0 ; x_{1}\right) \\
& \left.\left.-4 H\left(1,1,-1 ; x_{1}\right)-12 H\left(1,1,0 ; x_{1}\right)\right)+\mathcal{O}\left(\left(1-x_{2}\right)\right)\right]+\mathcal{O}\left(\epsilon^{2}\right),
\end{aligned}
$$

$$
\begin{aligned}
I_{17}= & \left(s_{12}\right)^{-3-2 \epsilon} S_{\Gamma} \frac{\left(1-x_{1}\right)^{-2 \epsilon}\left(1-x_{2}\right)^{-2 \epsilon}}{x_{1}\left(1-x_{1}\right)}[ \\
& -\frac{1}{2 \epsilon^{2}}+\frac{1}{\epsilon}\left(\log 2-H\left(-1 ; x_{1}\right)+H\left(0 ; x_{1}\right)\right)+\left(-\frac{\pi^{2}}{6}-2 \log ^{2} 2+\log 2\left(H\left(-1 ; x_{1}\right)\right.\right.
\end{aligned}
$$$$
\left.-2 H\left(0 ; x_{1}\right)-H\left(1 ; x_{1}\right)\right)-H\left(-1,-1 ; x_{1}\right)+H\left(-1,0 ; x_{1}\right)
$$$$
\left.+2 H\left(0,-1 ; x_{1}\right)-2 H\left(0,0 ; x_{1}\right)+H\left(1,-1 ; x_{1}\right)-H\left(1,0 ; x_{1}\right)\right)
$$$$
+\epsilon\left(\zeta_{3}+\frac{2}{3} \log ^{3} 2+\frac{1}{2} \log ^{2} 2\left(-H\left(-1 ; x_{1}\right)+4 H\left(0 ; x_{1}\right)+3 H\left(1 ; x_{1}\right)\right)\right.
$$$$
+\log 2\left(H\left(-1,-1 ; x_{1}\right)-H\left(-1,0 ; x_{1}\right)-2 H\left(0,-1 ; x_{1}\right)+4 H\left(0,0 ; x_{1}\right)+2 H\left(0,1 ; x_{1}\right)\right.
$$$$
\left.-H\left(1,-1 ; x_{1}\right)+3 H\left(1,0 ; x_{1}\right)+2 H\left(1,1 ; x_{1}\right)\right)+\frac{\pi^{2}}{3}\left(H\left(-1 ; x_{1}\right)+H\left(1 ; x_{1}\right)\right)
$$$$
-H\left(-1,-1,-1 ; x_{1}\right)+H\left(-1,-1,0 ; x_{1}\right)+H\left(-1,0,-1 ; x_{1}\right)-H\left(-1,0,0 ; x_{1}\right)
$$$$
+2 H\left(0,-1,-1 ; x_{1}\right)-2 H\left(0,-1,0 ; x_{1}\right)-4 H\left(0,0,-1 ; x_{1}\right)+4 H\left(0,0,0 ; x_{1}\right)
$$$$
-2 H\left(0,1,-1 ; x_{1}\right)+2 H\left(0,1,0 ; x_{1}\right)+H\left(1,-1,-1 ; x_{1}\right)-H\left(1,-1,0 ; x_{1}\right)
$$$$
\left.-3 H\left(1,0,-1 ; x_{1}\right)+3 H\left(1,0,0 ; x_{1}\right)-2 H\left(1,1,-1 ; x_{1}\right)+2 H\left(1,1,0 ; x_{1}\right)\right)
$$

$$
\begin{aligned}
& \left.+\mathcal{O}\left(\left(1-x_{2}\right)\right)\right]+\mathcal{O}\left(\epsilon^{2}\right), \\
I_{22}= & \left(s_{12}\right)^{-3-2 \epsilon} S_{\Gamma} \frac{\left(1-x_{1}\right)^{-2 \epsilon}\left(1-x_{2}\right)^{-2 \epsilon}}{x_{1}^{2}}[ \\
& \frac{1}{2 \epsilon^{2}}-\frac{1}{\epsilon} H\left(0 ; x_{1}\right)+\left(-\frac{1}{2} \log ^{2} 2-H\left(-1,0 ; x_{1}\right)+2 H\left(0,0 ; x_{1}\right)\right.
\end{aligned}
$$




$$
\begin{aligned}
& \left.-H\left(1 ; x_{1}\right) \log 2+H\left(1,-1 ; x_{1}\right)+H\left(1,0 ; x_{1}\right)\right) \\
& +\epsilon\left(-\zeta_{3}+\frac{1}{2} \log ^{3} 2+\frac{1}{2} \log ^{2} 2\left(2 H\left(0 ; x_{1}\right)+3 H\left(1 ; x_{1}\right)\right)\right. \\
& +\log 2\left(H\left(-1,0 ; x_{1}\right)+2 H\left(0,1 ; x_{1}\right)-H\left(1,-1 ; x_{1}\right)+H\left(1,0 ; x_{1}\right)+2 H\left(1,1 ; x_{1}\right)\right) \\
& +\frac{\pi^{2}}{3}\left(H\left(-1 ; x_{1}\right)-H\left(1 ; x_{1}\right)\right)-H\left(-1,-1,0 ; x_{1}\right)-H\left(-1,0,-1 ; x_{1}\right) \\
& +3 H\left(-1,0,0 ; x_{1}\right)+2 H\left(-1,1,0 ; x_{1}\right)+2 H\left(0,-1,0 ; x_{1}\right)-4 H\left(0,0,0 ; x_{1}\right) \\
& -2 H\left(0,1,-1 ; x_{1}\right)-2 H\left(0,1,0 ; x_{1}\right)+H\left(1,-1,-1 ; x_{1}\right)+H\left(1,-1,0 ; x_{1}\right) \\
& -H\left(1,0,-1 ; x_{1}\right)-3 H\left(1,0,0 ; x_{1}\right)-2 H\left(1,1,-1 ; x_{1}\right) \\
& \left.\left.-2 H\left(1,1,0 ; x_{1}\right)\right)+\mathcal{O}\left(\left(1-x_{2}\right)\right)\right]+\mathcal{O}\left(\epsilon^{2}\right) \\
& I_{23}=\mathcal{O}\left(\left(1-x_{2}\right)\right) \text {, } \\
& I_{24}=-\left(s_{12}\right)^{-3-2 \epsilon} S_{\Gamma} \frac{\left(1-x_{1}\right)^{-1-2 \epsilon}\left(1-x_{2}\right)^{-2 \epsilon}}{x_{1}}[ \\
& \frac{1}{\epsilon}\left(\log 2-H\left(-1 ; x_{1}\right)\right)+\left(-\frac{3}{2} \log ^{2} 2+\log 2\left(H\left(-1 ; x_{1}\right)-2 H\left(0 ; x_{1}\right)-2 H\left(1 ; x_{1}\right)\right)\right. \\
& \left.+2 H\left(0,-1 ; x_{1}\right)-H\left(-1,-1 ; x_{1}\right)+2 H\left(1,-1 ; x_{1}\right)\right) \\
& +\epsilon\left(\frac{7}{6} \log ^{3} 2+\frac{1}{2} \log ^{2} 2\left(-H\left(-1 ; x_{1}\right)+6 H\left(0 ; x_{1}\right)+6 H\left(1 ; x_{1}\right)\right)+\log 2\left(H\left(-1,-1 ; x_{1}\right)\right.\right. \\
& -2 H\left(0,-1 ; x_{1}\right)+4 H\left(0,0 ; x_{1}\right)+4 H\left(0,1 ; x_{1}\right)-2 H\left(1,-1 ; x_{1}\right)+4 H\left(1,0 ; x_{1}\right) \\
& \left.+4 H\left(1,1 ; x_{1}\right)\right)+\frac{2 \pi^{2}}{3} H\left(-1 ; x_{1}\right)-H\left(-1,-1,-1 ; x_{1}\right)+2 H\left(-1,0,0 ; x_{1}\right) \\
& +2 H\left(-1,1,0 ; x_{1}\right)+2 H\left(0,-1,-1 ; x_{1}\right)-4 H\left(0,0,-1 ; x_{1}\right)-4 H\left(0,1,-1 ; x_{1}\right) \\
& \left.+2 H\left(1,-1,-1 ; x_{1}\right)-4 H\left(1,0,-1 ; x_{1}\right)-4 H\left(1,1,-1 ; x_{1}\right)\right) \\
& \left.+\mathcal{O}\left(\left(1-x_{2}\right)\right)\right]+\mathcal{O}\left(\epsilon^{2}\right) \\
& I_{25}=\mathcal{O}\left(\left(1-x_{2}\right)\right) \text {, } \\
& I_{27}=\left(s_{12}\right)^{-3-2 \epsilon} S_{\Gamma} \frac{\left(1-x_{1}\right)^{-2 \epsilon}\left(1-x_{2}\right)^{-2 \epsilon}}{\left(1-x_{1}^{2}\right)}[ \\
& -\frac{1}{\epsilon^{2}}+\left(\log ^{2} 2+2 \log 2\left(H\left(0 ; x_{1}\right)+H\left(1 ; x_{1}\right)\right)+\frac{2 \pi^{2}}{3}-2 H\left(0,-1 ; x_{1}\right)+2 H\left(0,0 ; x_{1}\right)\right. \\
& \left.-2 H\left(1,-1 ; x_{1}\right)+2 H\left(1,0 ; x_{1}\right)\right)+\epsilon\left(6 \zeta_{3}-\log ^{3} 2-3 \log ^{2} 2\left(H\left(0 ; x_{1}\right)+H\left(1 ; x_{1}\right)\right)\right. \\
& +\log 2\left(2 H\left(0,-1 ; x_{1}\right)-6 H\left(0,0 ; x_{1}\right)-4 H\left(0,1 ; x_{1}\right)+2 H\left(1,-1 ; x_{1}\right)\right. \\
& \left.-6 H\left(1,0 ; x_{1}\right)-4 H\left(1,1 ; x_{1}\right)\right) \log 2-\frac{2 \pi^{2}}{3}\left(\log 2+H\left(0 ; x_{1}\right)+H\left(1 ; x_{1}\right)\right) \\
& -2 H\left(0,-1,-1 ; x_{1}\right)+2 H\left(0,-1,0 ; x_{1}\right)+6 H\left(0,0,-1 ; x_{1}\right)-6 H\left(0,0,0 ; x_{1}\right)
\end{aligned}
$$




$$
\begin{aligned}
& +4 H\left(0,1,-1 ; x_{1}\right)-4 H\left(0,1,0 ; x_{1}\right)-2 H\left(1,-1,-1 ; x_{1}\right)+2 H\left(1,-1,0 ; x_{1}\right) \\
& \left.+6 H\left(1,0,-1 ; x_{1}\right)-6 H\left(1,0,0 ; x_{1}\right)+4 H\left(1,1,-1 ; x_{1}\right)-4 H\left(1,1,0 ; x_{1}\right)\right) \\
& \left.+\mathcal{O}\left(\left(1-x_{2}\right)\right)\right]+\mathcal{O}\left(\epsilon^{2}\right) \\
& I_{28}=\left(s_{12}\right)^{-3-2 \epsilon} S_{\Gamma} \frac{\left(1-x_{1}\right)^{-2 \epsilon}\left(1-x_{2}\right)^{-2 \epsilon}}{x_{1}\left(1-x_{1}\right)}[ \\
& -\frac{1}{2 \epsilon^{2}}+\frac{1}{\epsilon}\left(\log 2-H\left(-1 ; x_{1}\right)+H\left(0 ; x_{1}\right)\right)+\left(-2 \log ^{2} 2+\log 2\left(H\left(-1 ; x_{1}\right)\right.\right. \\
& \left.-2 H\left(0 ; x_{1}\right)-H\left(1 ; x_{1}\right)\right)-H\left(-1,-1 ; x_{1}\right)+H\left(-1,0 ; x_{1}\right) \\
& \left.+2 H\left(0,-1 ; x_{1}\right)-2 H\left(0,0 ; x_{1}\right)+H\left(1,-1 ; x_{1}\right)-H\left(1,0 ; x_{1}\right)\right) \\
& +\epsilon\left(\zeta_{3}+\frac{2}{3} \log ^{3} 2+\frac{1}{2} \log ^{2} 2\left(-H\left(-1 ; x_{1}\right)+4 H\left(0 ; x_{1}\right)+3 H\left(1 ; x_{1}\right)\right)\right. \\
& +\log 2\left(H\left(-1,-1 ; x_{1}\right)-H\left(-1,0 ; x_{1}\right)-2 H\left(0,-1 ; x_{1}\right)\right. \\
& \left.+4 H\left(0,0 ; x_{1}\right)+2 H\left(0,1 ; x_{1}\right)-H\left(1,-1 ; x_{1}\right)+3 H\left(1,0 ; x_{1}\right)+2 H\left(1,1 ; x_{1}\right)\right) \\
& +\frac{\pi^{2}}{3}\left(H\left(-1 ; x_{1}\right)+H\left(1 ; x_{1}\right)\right)-H\left(-1,-1,-1 ; x_{1}\right)+H\left(-1,-1,0 ; x_{1}\right) \\
& +H\left(-1,0,-1 ; x_{1}\right)-H\left(-1,0,0 ; x_{1}\right)+2 H\left(0,-1,-1 ; x_{1}\right)-2 H\left(0,-1,0 ; x_{1}\right) \\
& -4 H\left(0,0,-1 ; x_{1}\right)+4 H\left(0,0,0 ; x_{1}\right)-2 H\left(0,1,-1 ; x_{1}\right)+2 H\left(0,1,0 ; x_{1}\right) \\
& +H\left(1,-1,-1 ; x_{1}\right)-H\left(1,-1,0 ; x_{1}\right)-3 H\left(1,0,-1 ; x_{1}\right)+3 H\left(1,0,0 ; x_{1}\right) \\
& \left.\left.-2 H\left(1,1,-1 ; x_{1}\right)+2 H\left(1,1,0 ; x_{1}\right)\right)+\mathcal{O}\left(\left(1-x_{2}\right)\right)\right] \\
& +\left(s_{12}\right)^{-3-2 \epsilon} S_{\Gamma} \frac{\left(1-x_{1}\right)^{-2 \epsilon}\left(1-x_{2}\right)^{-2 \epsilon}}{1-x_{1}^{2}}[ \\
& -\frac{1}{\epsilon^{2}}+\left(\log ^{2} 2+\frac{2 \pi^{2}}{3}+\log 2\left(2 H\left(0 ; x_{1}\right)+2 H\left(1 ; x_{1}\right)\right)-2 H\left(0,-1 ; x_{1}\right)\right. \\
& \left.+2 H\left(0,0 ; x_{1}\right)-2 H\left(1,-1 ; x_{1}\right)+2 H\left(1,0 ; x_{1}\right)\right) \\
& +\epsilon\left(6 \zeta_{3}-\log ^{3} 2-3 \log ^{2} 2\left(H\left(0 ; x_{1}\right)+H\left(1 ; x_{1}\right)\right)+\log 2\left(2 H\left(0,-1 ; x_{1}\right)\right.\right. \\
& \left.-6 H\left(0,0 ; x_{1}\right)-4 H\left(0,1 ; x_{1}\right)+2 H\left(1,-1 ; x_{1}\right)-6 H\left(1,0 ; x_{1}\right)-4 H\left(1,1 ; x_{1}\right)\right) \\
& +\frac{\pi^{2}}{3}\left(-2 \log 2-2 H\left(0 ; x_{1}\right)-2 H\left(1 ; x_{1}\right)\right)-2 H\left(0,-1,-1 ; x_{1}\right)+2 H\left(0,-1,0 ; x_{1}\right) \\
& +6 H\left(0,0,-1 ; x_{1}\right)-6 H\left(0,0,0 ; x_{1}\right)+4 H\left(0,1,-1 ; x_{1}\right)-4 H\left(0,1,0 ; x_{1}\right) \\
& -2 H\left(1,-1,-1 ; x_{1}\right)+2 H\left(1,-1,0 ; x_{1}\right)+6 H\left(1,0,-1 ; x_{1}\right)-6 H\left(1,0,0 ; x_{1}\right) \\
& \left.\left.+4 H\left(1,1,-1 ; x_{1}\right)-4 H\left(1,1,0 ; x_{1}\right)\right)+\mathcal{O}\left(\left(1-x_{2}\right)\right)\right]+\mathcal{O}\left(\epsilon^{2}\right) \text {, }
\end{aligned}
$$




$$
\begin{aligned}
I_{29}= & \left(s_{12}\right)^{-3-2 \epsilon} S_{\Gamma} \frac{\left(1-x_{1}\right)^{-1-2 \epsilon}\left(1-x_{2}\right)^{-1-2 \epsilon}}{2 x_{1}}[ \\
& \frac{3}{\epsilon^{2}}+\frac{1}{\epsilon}\left(-4 \log 2+4 H\left(-1 ; x_{1}\right)-4 H\left(0 ; x_{1}\right)\right)+\left(2 \log ^{2} 2-\frac{4 \pi^{2}}{3}\right. \\
& +\log 2\left(-4 H\left(-1 ; x_{1}\right)+4 H\left(0 ; x_{1}\right)\right)+4 H\left(-1,-1 ; x_{1}\right)-4 H\left(-1,0 ; x_{1}\right) \\
& \left.-4 H\left(0,-1 ; x_{1}\right)+4 H\left(0,0 ; x_{1}\right)\right)+\epsilon\left(-14 \zeta_{3}+\frac{8 \pi^{2}}{3}\left(\log 2-H\left(-1 ; x_{1}\right)+H\left(0 ; x_{1}\right)\right)\right. \\
& +4 \log 2\left(-H\left(-1,-1 ; x_{1}\right)-H\left(-1,1 ; x_{1}\right)+H\left(0,-1 ; x_{1}\right)+H\left(0,1 ; x_{1}\right)\right) \\
& +4 H\left(-1,-1,-1 ; x_{1}\right)-4 H\left(-1,-1,0 ; x_{1}\right)+4 H\left(-1,1,-1 ; x_{1}\right)-4 H\left(-1,1,0 ; x_{1}\right) \\
& \left.-4 H\left(0,-1,-1 ; x_{1}\right)+4 H\left(0,-1,0 ; x_{1}\right)-4 H\left(0,1,-1 ; x_{1}\right)+4 H\left(0,1,0 ; x_{1}\right)\right) \\
& \left.+\mathcal{O}\left(\left(1-x_{2}\right)\right)\right]+\mathcal{O}\left(\epsilon^{2}\right)
\end{aligned}
$$

\section{A.3 Collinear $x_{2}$ region}

In the collinear $x_{2}$ region, we can expand around $x_{1}=1$, and the master integrals read:

$$
\begin{aligned}
& I_{13}=\left(s_{12}\right)^{-3-2 \epsilon} S_{\Gamma} \frac{\left(1-x_{1}\right)^{-2 \epsilon}\left(1-x_{2}\right)^{-2 \epsilon}}{1+2 x_{2}}[ \\
& \frac{1}{\epsilon}\left(-\log 2+H\left(-1 ; x_{2}\right)-2 H\left(0 ; x_{2}\right)\right) \\
& +\left(\frac{3}{2} \log ^{2} 2+\log 2\left(-H\left(-1 ; x_{2}\right)+4 H\left(0 ; x_{2}\right)+2 H\left(1 ; x_{2}\right)\right)+\frac{2 \pi^{2}}{3}+H\left(-1,-1 ; x_{2}\right)\right. \\
& \left.-2 H\left(-1,0 ; x_{2}\right)-4 H\left(0,-1 ; x_{2}\right)+6 H\left(0,0 ; x_{2}\right)-2 H\left(1,-1 ; x_{2}\right)+4 H\left(1,0 ; x_{2}\right)\right) \\
& +\epsilon\left(4 \zeta_{3}-\frac{7}{6} \log ^{3} 2+\log ^{2} 2\left(\frac{1}{2} H\left(-1 ; x_{2}\right)-4 H\left(0 ; x_{2}\right)-3 H\left(1 ; x_{2}\right)\right)\right. \\
& +\log 2\left(-H\left(-1,-1 ; x_{2}\right)+2 H\left(-1,0 ; x_{2}\right)+4 H\left(0,-1 ; x_{2}\right)-10 H\left(0,0 ; x_{2}\right)\right. \\
& \left.-4 H\left(0,1 ; x_{2}\right)+2 H\left(1,-1 ; x_{2}\right)-8 H\left(1,0 ; x_{2}\right)-4 H\left(1,1 ; x_{2}\right)\right) \\
& -\frac{2 \pi^{2}}{3}\left(\log 2+H\left(0 ; x_{2}\right)+2 H\left(1 ; x_{2}\right)\right)+H\left(-1,-1,-1 ; x_{2}\right)-2 H\left(-1,-1,0 ; x_{2}\right) \\
& -2 H\left(-1,0,-1 ; x_{2}\right)+4 H\left(-1,0,0 ; x_{2}\right)+2 H\left(-1,1,0 ; x_{2}\right)-4 H\left(0,-1,-1 ; x_{2}\right) \\
& +6 H\left(0,-1,0 ; x_{2}\right)+10 H\left(0,0,-1 ; x_{2}\right)-14 H\left(0,0,0 ; x_{2}\right)+4 H\left(0,1,-1 ; x_{2}\right) \\
& -8 H\left(0,1,0 ; x_{2}\right)-2 H\left(1,-1,-1 ; x_{2}\right)+4 H\left(1,-1,0 ; x_{2}\right)+8 H\left(1,0,-1 ; x_{2}\right) \\
& \left.-12 H\left(1,0,0 ; x_{2}\right)+4 H\left(1,1,-1 ; x_{2}\right)-8 H\left(1,1,0 ; x_{2}\right)\right) \\
& \left.+\mathcal{O}\left(\left(1-x_{1}\right)\right)\right]+\mathcal{O}\left(\epsilon^{2}\right) \\
& I_{16}=\left(s_{12}\right)^{-3-2 \epsilon} S_{\Gamma} \frac{\left(1-x_{1}\right)^{-2 \epsilon}\left(1-x_{2}\right)^{-2 \epsilon}}{1-x_{2}^{2}}[
\end{aligned}
$$




$$
\begin{aligned}
& -\frac{1}{\epsilon^{2}}+\frac{1}{\epsilon}\left(\log 2-H\left(-1 ; x_{2}\right)\right)+\left(-\frac{1}{2} \log ^{2} 2+H\left(-1 ; x_{2}\right) \log 2+\frac{2 \pi^{2}}{3}\right. \\
& \left.-H\left(-1,-1 ; x_{2}\right)+2 H\left(0,0 ; x_{2}\right)+2 H\left(1,0 ; x_{2}\right)\right)+\epsilon\left(6 \zeta_{3}+\frac{1}{6} \log ^{3} 2\right. \\
& -\frac{1}{2} \log ^{2} 2 H\left(-1 ; x_{2}\right)+\log 2\left(H\left(-1,-1 ; x_{2}\right)-2 H\left(0,0 ; x_{2}\right)-2 H\left(1,0 ; x_{2}\right)\right) \\
& +\frac{2 \pi^{2}}{3}\left(-\log 2+H\left(-1 ; x_{2}\right)-H\left(0 ; x_{2}\right)-H\left(1 ; x_{2}\right)\right)-H\left(-1,-1,-1 ; x_{2}\right) \\
& +2 H\left(-1,0,0 ; x_{2}\right)+2 H\left(-1,1,0 ; x_{2}\right)+2 H\left(0,-1,0 ; x_{2}\right)+2 H\left(0,0,-1 ; x_{2}\right) \\
& -6 H\left(0,0,0 ; x_{2}\right)-4 H\left(0,1,0 ; x_{2}\right)+2 H\left(1,-1,0 ; x_{2}\right)+2 H\left(1,0,-1 ; x_{2}\right) \\
& \left.\left.-6 H\left(1,0,0 ; x_{2}\right)-4 H\left(1,1,0 ; x_{2}\right)\right)+\mathcal{O}\left(\left(1-x_{1}\right)\right)\right]+\mathcal{O}\left(\epsilon^{2}\right), \\
& I_{17}=\left(s_{12}\right)^{-3-2 \epsilon} S_{\Gamma} \frac{\left(1-x_{1}\right)^{-1-2 \epsilon}\left(1-x_{2}\right)^{-2 \epsilon}}{2 x_{2}^{2}}[ \\
& -\frac{1}{\epsilon^{2}}+\frac{2}{\epsilon} H\left(0 ; x_{2}\right)+\left(2 \log ^{2} 2+4 H\left(1 ; x_{2}\right) \log 2-\frac{\pi^{2}}{3}-4 H\left(0,0 ; x_{2}\right)-4 H\left(1,-1 ; x_{2}\right)\right. \\
& \left.-2 H\left(1,0 ; x_{2}\right)\right)+\epsilon\left(-4 \zeta_{3}-\frac{8}{3} \log ^{3} 2+\log ^{2} 2\left(-4 H\left(0 ; x_{2}\right)-8 H\left(1 ; x_{2}\right)\right)\right. \\
& +\log 2\left(-8 H\left(0,1 ; x_{2}\right)+4 H\left(1,-1 ; x_{2}\right)-4 H\left(1,0 ; x_{2}\right)-12 H\left(1,1 ; x_{2}\right)\right) \\
& +\frac{\pi^{2}}{3}\left(2 \log 2+2 H\left(0 ; x_{2}\right)+5 H\left(1 ; x_{2}\right)\right)+8 H\left(0,0,0 ; x_{2}\right)+8 H\left(0,1,-1 ; x_{2}\right) \\
& +4 H\left(0,1,0 ; x_{2}\right)-4 H\left(1,-1,-1 ; x_{2}\right)+4 H\left(1,-1,0 ; x_{2}\right)+4 H\left(1,0,-1 ; x_{2}\right) \\
& \left.+6 H\left(1,0,0 ; x_{2}\right)+12 H\left(1,1,-1 ; x_{2}\right)+4 H\left(1,1,0 ; x_{2}\right)\right) \\
& \left.+\mathcal{O}\left(\left(1-x_{1}\right)\right)\right]+\mathcal{O}\left(\epsilon^{2}\right) \\
& M_{22}=\left(s_{12}\right)^{-3-2 \epsilon} S_{\Gamma} \frac{\left(1-x_{1}\right)^{-2 \epsilon}\left(1-x_{2}\right)^{-2 \epsilon}}{x_{2}}\left[\frac{1}{2 \epsilon^{2}}+\frac{1}{2 \epsilon}\left(-\log 2+H\left(-1 ; x_{2}\right)\right)\right. \\
& +\frac{1}{4} \log ^{2} 2-\frac{1}{2} H\left(-1 ; x_{2}\right) \log 2-\frac{\pi^{2}}{3}+\frac{1}{2} H\left(-1,-1 ; x_{2}\right)-H\left(0,0 ; x_{2}\right)-H\left(1,0 ; x_{2}\right) \\
& +\epsilon\left(-3 \zeta_{3}-\frac{1}{12} \log ^{3} 2+\frac{1}{4} \log ^{2} 2 H\left(-1 ; x_{2}\right)\right. \\
& +\log 2\left(-\frac{1}{2} H\left(-1,-1 ; x_{2}\right)+H\left(0,0 ; x_{2}\right)+H\left(1,0 ; x_{2}\right)\right) \\
& +\frac{\pi^{2}}{3}\left(\log 2-H\left(-1 ; x_{2}\right)+H\left(1 ; x_{2}\right)+H\left(0 ; x_{2}\right)\right)+\frac{1}{2} H\left(-1,-1,-1 ; x_{2}\right) \\
& -H\left(-1,0,0 ; x_{2}\right)-H\left(-1,1,0 ; x_{2}\right)-H\left(0,-1,0 ; x_{2}\right)-H\left(0,0,-1 ; x_{2}\right) \\
& +3 H\left(0,0,0 ; x_{2}\right)+2 H\left(0,1,0 ; x_{2}\right)-H\left(1,-1,0 ; x_{2}\right)-H\left(1,0,-1 ; x_{2}\right) \\
& \left.\left.+3 H\left(1,0,0 ; x_{2}\right)+2 H\left(1,1,0 ; x_{2}\right)\right)+\mathcal{O}\left(\left(1-x_{1}\right)\right)\right]+\mathcal{O}\left(\epsilon^{2}\right),
\end{aligned}
$$




$$
\begin{aligned}
& I_{23}=\left(s_{12}\right)^{-3-2 \epsilon} S_{\Gamma} \frac{\left(1-x_{1}\right)^{-1-2 \epsilon}\left(1-x_{2}\right)^{-2 \epsilon}}{2 x_{2}\left(1+x_{2}\right)}[ \\
& \frac{2}{\epsilon} H\left(0 ; x_{2}\right)-\frac{4 \pi^{2}}{3}-4 H\left(0 ; x_{2}\right) \log 2-4 H\left(-1,0 ; x_{2}\right)+4 H\left(0,-1 ; x_{2}\right)-6 H\left(0,0 ; x_{2}\right) \\
& \left.-4 H\left(1,0 ; x_{2}\right)+4 g_{23 b}\left(x_{2}\right)+\mathcal{O}\left(\left(1-x_{1}\right)\right)\right]+\mathcal{O}(\epsilon), \\
& I_{24}=\left(s_{12}\right)^{-3-2 \epsilon} S_{\Gamma} \frac{\left(1-x_{1}\right)^{-2 \epsilon}\left(1-x_{2}\right)^{-1-2 \epsilon}}{x_{2}}[ \\
& \frac{1}{\epsilon}\left(-\log 2+H\left(-1 ; x_{2}\right)\right)+\left(\frac{3}{2} \log ^{2} 2-\frac{\pi^{2}}{6}+\log 2\left(-H\left(-1 ; x_{2}\right)+2 H\left(1 ; x_{2}\right)\right)\right. \\
& \left.+H\left(-1,-1 ; x_{2}\right)-2 H\left(1,-1 ; x_{2}\right)\right)+\epsilon\left(-\frac{13}{4} \zeta_{3}-\frac{7}{6} \log ^{3} 2\right. \\
& +\frac{1}{2} \log ^{2} 2\left(H\left(-1 ; x_{2}\right)-6 H\left(1 ; x_{2}\right)\right)+\log 2\left(2 H\left(1,0 ; x_{2}\right)+2 H\left(0,0 ; x_{2}\right)-4 H\left(1,1 ; x_{2}\right)\right. \\
& \left.+2 H\left(1,-1 ; x_{2}\right)-H\left(-1,-1 ; x_{2}\right)\right)+\frac{\pi^{2}}{3}\left(3 \log 2-2 H\left(-1 ; x_{2}\right)+H\left(1 ; x_{2}\right)\right) \\
& +H\left(-1,-1,-1 ; x_{2}\right)-2 H\left(-1,0,0 ; x_{2}\right)-2 H\left(-1,1,0 ; x_{2}\right)-2 H\left(0,-1,0 ; x_{2}\right) \\
& -2 H\left(0,0,-1 ; x_{2}\right)-2 H\left(1,-1,-1 ; x_{2}\right)-2 H\left(1,-1,0 ; x_{2}\right)-2 H\left(1,0,-1 ; x_{2}\right) \\
& \left.\left.+4 H\left(1,1,-1 ; x_{2}\right)\right)+\mathcal{O}\left(\left(1-x_{1}\right)\right)\right]+\mathcal{O}\left(\epsilon^{2}\right), \\
& I_{25}=\left(s_{12}\right)^{-3-2 \epsilon} S_{\Gamma} \frac{\left(1-x_{1}\right)^{-1-2 \epsilon}\left(1-x_{2}\right)^{-2 \epsilon}}{2 x_{2}}[ \\
& \frac{2}{\epsilon} H\left(0 ; x_{2}\right)-\pi^{2}-4 H\left(0 ; x_{2}\right) \log 2+4 H\left(0,-1 ; x_{2}\right)-8 H\left(0,0 ; x_{2}\right) \\
& \left.-4 H\left(1,0 ; x_{2}\right)+4 g_{23 b}\left(x_{2}\right)+\mathcal{O}\left(\left(1-x_{1}\right)\right)\right]+\mathcal{O}(\epsilon), \\
& I_{27}=\left(s_{12}\right)^{-3-2 \epsilon} S_{\Gamma} \frac{\left(1-x_{1}\right)^{-1-2 \epsilon}\left(1-x_{2}\right)^{-2 \epsilon}}{2 x_{2}}[ \\
& -\frac{1}{\epsilon^{2}}+\frac{1}{\epsilon}\left(2 \log 2-2 H\left(-1 ; x_{2}\right)\right)+\left(-2 \log ^{2} 2-4 \log 2 H\left(1 ; x_{2}\right)+\pi^{2}+2 H\left(0,0 ; x_{2}\right)\right. \\
& \left.+4 H\left(1,-1 ; x_{2}\right)+2 H\left(1,0 ; x_{2}\right)\right)+\epsilon\left(\frac{25}{2} \zeta_{3}+\frac{4}{3} \log ^{3} 2+\log ^{2} 2\left(2 H\left(-1 ; x_{2}\right)\right.\right. \\
& \left.+6 H\left(1 ; x_{2}\right)\right)+\log 2\left(4 H\left(-1,0 ; x_{2}\right)+4 H\left(-1,1 ; x_{2}\right)-4 H\left(0,0 ; x_{2}\right)+12 H\left(1,1 ; x_{2}\right)\right) \\
& +\frac{\pi^{2}}{3}\left(-6 \log 2+4 H\left(-1 ; x_{2}\right)-3 H\left(0 ; x_{2}\right)-5 H\left(1 ; x_{2}\right)\right)-4 H\left(-1,0,-1 ; x_{2}\right) \\
& +4 H\left(-1,0,0 ; x_{2}\right)-4 H\left(-1,1,-1 ; x_{2}\right)+4 H\left(-1,1,0 ; x_{2}\right)+4 H\left(0,0,-1 ; x_{2}\right) \\
& -6 H\left(0,0,0 ; x_{2}\right)-4 H\left(0,1,0 ; x_{2}\right)-6 H\left(1,0,0 ; x_{2}\right)-12 H\left(1,1,-1 ; x_{2}\right) \\
& \left.\left.-4 H\left(1,1,0 ; x_{2}\right)\right)+\mathcal{O}\left(\left(1-x_{1}\right)\right)\right]+\mathcal{O}\left(\epsilon^{2}\right),
\end{aligned}
$$




$$
\begin{aligned}
& I_{28}=\left(s_{12}\right)^{-3-2 \epsilon} S_{\Gamma} \frac{\left(1-x_{1}\right)^{-1-2 \epsilon}\left(1-x_{2}\right)^{-2 \epsilon}}{2 x_{2}}[ \\
& -\frac{2}{\epsilon^{2}}+\frac{1}{\epsilon}\left(2 \log 2-2 H\left(-1 ; x_{2}\right)+2 H\left(0 ; x_{2}\right)\right)+\frac{2 \pi^{2}}{3}+\epsilon\left(4 \zeta_{3}-\frac{4}{3} \log ^{3} 2\right. \\
& +\log ^{2} 2\left(2 H\left(-1 ; x_{2}\right)-4 H\left(0 ; x_{2}\right)-2 H\left(1 ; x_{2}\right)\right)+\log 2\left(4 H\left(-1,0 ; x_{2}\right)\right. \\
& +4 H\left(-1,1 ; x_{2}\right)+4 H\left(0,-1 ; x_{2}\right)-8 H\left(0,0 ; x_{2}\right)-4 H\left(0,1 ; x_{2}\right)+4 H\left(1,-1 ; x_{2}\right) \\
& \left.-4 H\left(1,0 ; x_{2}\right)\right)+\frac{4 \pi^{2}}{3}\left(-\log 2+H\left(-1 ; x_{2}\right)-H\left(0 ; x_{2}\right)\right)-4 H\left(-1,0,-1 ; x_{2}\right) \\
& +4 H\left(-1,0,0 ; x_{2}\right)-4 H\left(-1,1,-1 ; x_{2}\right)+4 H\left(-1,1,0 ; x_{2}\right)-4 H\left(0,-1,-1 ; x_{2}\right) \\
& +4 H\left(0,-1,0 ; x_{2}\right)+8 H\left(0,0,-1 ; x_{2}\right)-8 H\left(0,0,0 ; x_{2}\right)+4 H\left(0,1,-1 ; x_{2}\right) \\
& -4 H\left(0,1,0 ; x_{2}\right)-4 H\left(1,-1,-1 ; x_{2}\right)+4 H\left(1,-1,0 ; x_{2}\right)+4 H\left(1,0,-1 ; x_{2}\right) \\
& \left.\left.-4 H\left(1,0,0 ; x_{2}\right)\right)+\mathcal{O}\left(\left(1-x_{1}\right)\right)\right]+\mathcal{O}\left(\epsilon^{2}\right) \\
& I_{29}=\left(s_{12}\right)^{-3-2 \epsilon} S_{\Gamma} \frac{\left(1-x_{1}\right)^{-1-2 \epsilon}\left(1-x_{2}\right)^{-1-2 \epsilon}}{2 x_{2}}[ \\
& \frac{3}{\epsilon^{2}}+\frac{1}{\epsilon}\left(-4 \log 2+4 H\left(-1 ; x_{2}\right)-4 H\left(0 ; x_{2}\right)\right)+\left(2 \log ^{2} 2-\frac{4 \pi^{2}}{3}\right. \\
& +\log 2\left(-4 H\left(-1 ; x_{2}\right)+4 H\left(0 ; x_{2}\right)\right)+4 H\left(-1,-1 ; x_{2}\right)-4 H\left(-1,0 ; x_{2}\right) \\
& \left.-4 H\left(0,-1 ; x_{2}\right)+4 H\left(0,0 ; x_{2}\right)\right)+\epsilon\left(-14 \zeta_{3}+\frac{8 \pi^{2}}{3}\left(\log 2-H\left(-1 ; x_{2}\right)+H\left(0 ; x_{2}\right)\right)\right. \\
& +\log 2\left(-4 H\left(-1,-1 ; x_{2}\right)-4 H\left(-1,1 ; x_{2}\right)+4 H\left(0,-1 ; x_{2}\right)+4 H\left(0,1 ; x_{2}\right)\right) \\
& +4 H\left(-1,-1,-1 ; x_{2}\right)-4 H\left(-1,-1,0 ; x_{2}\right)+4 H\left(-1,1,-1 ; x_{2}\right)-4 H\left(-1,1,0 ; x_{2}\right) \\
& \left.-4 H\left(0,-1,-1 ; x_{2}\right)+4 H\left(0,-1,0 ; x_{2}\right)-4 H\left(0,1,-1 ; x_{2}\right)+4 H\left(0,1,0 ; x_{2}\right)\right) \\
& \left.+\mathcal{O}\left(\left(1-x_{1}\right)\right)\right]+\mathcal{O}\left(\epsilon^{2}\right) \text {. }
\end{aligned}
$$

The combination $\left(1+x_{2}\right) I_{23}-I_{25}$ appears in this collinear limit to order $\mathcal{O}(\epsilon)$, it reads:

$$
\begin{aligned}
\left(1+x_{2}\right) I_{23}-I_{25}= & \left(s_{12}\right)^{-3-2 \epsilon} S_{\Gamma} \frac{\left(1-x_{1}\right)^{-1-2 \epsilon}\left(1-x_{2}\right)^{-2 \epsilon}}{2 x_{2}}[ \\
& -\frac{\pi^{2}}{3}-4 H\left(-1,0 ; x_{2}\right)+2 H\left(0,0 ; x_{2}\right) \\
& +\epsilon\left(-30 \zeta_{3}+\log 2\left(8 H\left(-1,0 ; x_{2}\right)-4 H\left(0,0 ; x_{2}\right)\right)\right. \\
& +\frac{2 \pi^{2}}{3}\left(\log 2+4 H\left(-1 ; x_{2}\right)-2 H\left(0 ; x_{2}\right)+H\left(1 ; x_{2}\right)\right) \\
& +8 H\left(-1,-1,0 ; x_{2}\right)-8 H\left(-1,0,-1 ; x_{2}\right)+12 H\left(-1,0,0 ; x_{2}\right) \\
& +8 H\left(-1,1,0 ; x_{2}\right)-4 H\left(0,-1,0 ; x_{2}\right)+4 H\left(0,0,-1 ; x_{2}\right)
\end{aligned}
$$




$$
\begin{aligned}
& -10 H\left(0,0,0 ; x_{2}\right)-4 H\left(0,1,0 ; x_{2}\right)+8 H\left(1,-1,0 ; x_{2}\right) \\
& \left.\left.-4 H\left(1,0,0 ; x_{2}\right)+8 g_{23 c}\left(x_{2}\right)\right)+\mathcal{O}\left(\left(1-x_{1}\right)\right)\right]+\mathcal{O}\left(\epsilon^{2}\right),
\end{aligned}
$$

\section{A.4 Soft region}

In the soft region, $x_{1} \rightarrow 1$ and $x_{2} \rightarrow 1$. The master integrals then become:

$$
\begin{aligned}
& I_{13}=\left(s_{12}\right)^{-2-2 \epsilon} S_{\Gamma} \frac{\left(1-x_{1}\right)^{-2 \epsilon}\left(1-x_{2}\right)^{1-2 \epsilon}}{2 \epsilon(1-2 \epsilon)}\left[1-\frac{\pi^{2}}{3} \epsilon^{2}-4 \zeta_{3} \epsilon^{3}-\frac{\pi^{4}}{45} \epsilon^{4}+\mathcal{O}\left(\epsilon^{5}\right)\right], \\
& I_{16}=-\left(s_{12}\right)^{-3-2 \epsilon} S_{\Gamma} \frac{\left(1-x_{1}\right)^{-2 \epsilon}\left(-1-x_{2}\right)^{1-2 \epsilon}}{2 \epsilon^{2}}\left[1-\frac{\pi^{2}}{3} \epsilon^{2}-4 \zeta_{3} \epsilon^{3}-\frac{\pi^{4}}{45} \epsilon^{4}+\mathcal{O}\left(\epsilon^{5}\right)\right], \\
& I_{17}=-\left(s_{12}\right)^{-3-2 \epsilon} S_{\Gamma} \frac{\left(1-x_{1}\right)^{-1-2 \epsilon}\left(1-x_{2}\right)^{-2 \epsilon}}{2 \epsilon^{2}}\left[1-\frac{\pi^{2}}{3} \epsilon^{2}-4 \zeta_{3} \epsilon^{3}-\frac{\pi^{4}}{45} \epsilon^{4}+\mathcal{O}\left(\epsilon^{5}\right)\right], \\
& I_{22}=\left(s_{12}\right)^{-3-2 \epsilon} S_{\Gamma} \frac{\left(1-x_{1}\right)^{-2 \epsilon}\left(1-x_{2}\right)^{-2 \epsilon}}{2 \epsilon^{2}}\left[1-\frac{\pi^{2}}{3} \epsilon^{2}-4 \zeta_{3} \epsilon^{3}-\frac{\pi^{4}}{45} \epsilon^{4}+\mathcal{O}\left(\epsilon^{5}\right)\right], \\
& I_{23}=-\left(s_{12}\right)^{-3-2 \epsilon} S_{\Gamma} \frac{\left(1-x_{1}\right)^{-1-2 \epsilon}\left(1-x_{2}\right)^{1-2 \epsilon}}{2 \epsilon(1-2 \epsilon)}\left[1-\frac{\pi^{2}}{3} \epsilon^{2}-4 \zeta_{3} \epsilon^{3}-\frac{\pi^{4}}{45} \epsilon^{4}+\mathcal{O}\left(\epsilon^{5}\right)\right], \\
& I_{24}=\left(s_{12}\right)^{-3-2 \epsilon} S_{\Gamma} \frac{\left(1-x_{1}\right)^{-2 \epsilon}\left(1-x_{2}\right)^{-2 \epsilon}}{2 \epsilon}\left[1-\frac{\pi^{2}}{3} \epsilon^{2}-4 \zeta_{3} \epsilon^{3}-\frac{\pi^{4}}{45} \epsilon^{4}+\mathcal{O}\left(\epsilon^{5}\right)\right], \\
& I_{25}=-\left(s_{12}\right)^{-3-2 \epsilon} S_{\Gamma} \frac{\left(1-x_{1}\right)^{-1-2 \epsilon}\left(1-x_{2}\right)^{1-2 \epsilon}}{\epsilon(1-2 \epsilon)}\left[1-\frac{\pi^{2}}{3} \epsilon^{2}-4 \zeta_{3} \epsilon^{3}-\frac{\pi^{4}}{45} \epsilon^{4}+\mathcal{O}\left(\epsilon^{5}\right)\right], \\
& I_{27}=-\left(s_{12}\right)^{-3-2 \epsilon} S_{\Gamma} \frac{\left(1-x_{1}\right)^{-1-2 \epsilon}\left(1-x_{2}\right)^{-2 \epsilon}}{2 \epsilon^{2}}\left[1-\frac{\pi^{2}}{3} \epsilon^{2}-4 \zeta_{3} \epsilon^{3}-\frac{\pi^{4}}{45} \epsilon^{4}+\mathcal{O}\left(\epsilon^{5}\right)\right], \\
& I_{28}=-\left(s_{12}\right)^{-3-2 \epsilon} S_{\Gamma} \frac{\left(1-x_{1}\right)^{-1-2 \epsilon}\left(1-x_{2}\right)^{-2 \epsilon}}{\epsilon^{2}}\left[1-\frac{\pi^{2}}{3} \epsilon^{2}-4 \zeta_{3} \epsilon^{3}-\frac{\pi^{4}}{45} \epsilon^{4}+\mathcal{O}\left(\epsilon^{5}\right)\right], \\
& I_{29}=\left(s_{12}\right)^{-3-2 \epsilon} S_{\Gamma} \frac{\left(1-x_{1}\right)^{-1-2 \epsilon}\left(1-x_{2}\right)^{-1-2 \epsilon}}{\epsilon^{2}}\left[\frac{3}{2}-\frac{2 \pi^{2}}{3} \epsilon^{2}-11 \zeta_{3} \epsilon^{3}-\frac{3 \pi^{4}}{20} \epsilon^{4}+\mathcal{O}\left(\epsilon^{5}\right)\right] .
\end{aligned}
$$

Open Access. This article is distributed under the terms of the Creative Commons Attribution License which permits any use, distribution and reproduction in any medium, provided the original author(s) and source are credited.

\section{References}

[1] R.K. Ellis, W.J. Stirling and B.R. Webber, QCD and Collider Physics, Cambridge University Press, Cambridge, U.K. (1996).

[2] G. Dissertori, I.G. Knowles and M. Schmelling, Quantum Chromodynamics: High Energy Experiments and Theory, Oxford University Press Oxford, U.K. (2003).

[3] Z. Bern, L.J. Dixon, D.C. Dunbar and D.A. Kosower, One loop $n$ point gauge theory amplitudes, unitarity and collinear limits, Nucl. Phys. B 425 (1994) 217 [hep-ph/9403226] [INSPIRE].

[4] D.A. Kosower, All order collinear behavior in gauge theories, Nucl. Phys. B 552 (1999) 319 [hep-ph/9901201] [INSPIRE]. 
[5] D.A. Kosower and P. Uwer, One loop splitting amplitudes in gauge theory, Nucl. Phys. B 563 (1999) 477 [hep-ph/9903515] [INSPIRE].

[6] Z. Bern, V. Del Duca and C.R. Schmidt, The infrared behavior of one loop gluon amplitudes at next-to-next-to-leading order, Phys. Lett. B 445 (1998) 168 [hep-ph/9810409] [INSPIRE].

[7] Z. Bern, V. Del Duca, W.B. Kilgore and C.R. Schmidt, The infrared behavior of one loop QCD amplitudes at next-to-next-to leading order, Phys. Rev. D 60 (1999) 116001 [hep-ph/9903516] [INSPIRE].

[8] S. Catani and M. Grazzini, The soft gluon current at one loop order, Nucl. Phys. B 591 (2000) 435 [hep-ph/0007142] [inSPIRE].

[9] D.A. Kosower, All orders singular emission in gauge theories, Phys. Rev. Lett. 91 (2003) 061602 [hep-ph/0301069] [INSPIRE].

[10] S. Weinzierl, Subtraction terms for one loop amplitudes with one unresolved parton, JHEP 07 (2003) 052 [hep-ph/0306248] [INSPIRE].

[11] C. Anastasiou, Z. Bern, L.J. Dixon and D. Kosower, Planar amplitudes in maximally supersymmetric Yang-Mills theory, Phys. Rev. Lett. 91 (2003) 251602 [hep-th/0309040] [INSPIRE].

[12] Z. Bern, L.J. Dixon and D.A. Kosower, Two-loop $g \rightarrow g$ splitting amplitudes in $Q C D$, JHEP 08 (2004) 012 [hep-ph/0404293] [INSPIRE].

[13] S. Badger and E.W.N. Glover, Two loop splitting functions in QCD, JHEP 07 (2004) 040 [hep-ph/0405236] [INSPIRE].

[14] A. Gehrmann-De Ridder and E.W.N. Glover, A Complete $O\left(\alpha \alpha_{s}\right)$ calculation of the photon +1 jet rate in $e^{+} e^{-}$annihilation, Nucl. Phys. B 517 (1998) 269 [hep-ph/9707224] [INSPIRE].

[15] J.M. Campbell and E.W.N. Glover, Double unresolved approximations to multiparton scattering amplitudes, Nucl. Phys. B 527 (1998) 264 [hep-ph/9710255] [INSPIRE].

[16] S. Catani and M. Grazzini, Collinear factorization and splitting functions for next-to-next-to-leading order QCD calculations, Phys. Lett. B 446 (1999) 143 [hep-ph/9810389] [INSPIRE].

[17] S. Catani and M. Grazzini, Infrared factorization of tree level QCD amplitudes at the next-to-next-to-leading order and beyond, Nucl. Phys. B 570 (2000) 287 [hep-ph/9908523] [INSPIRE].

[18] F.A. Berends and W.T. Giele, Multiple Soft Gluon Radiation in Parton Processes, Nucl. Phys. B 313 (1989) 595 [inSPIRE].

[19] V. Del Duca, A. Frizzo and F. Maltoni, Factorization of tree QCD amplitudes in the high-energy limit and in the collinear limit, Nucl. Phys. B 568 (2000) 211 [hep-ph/9909464] [INSPIRE].

[20] T. Birthwright, E.W.N. Glover, V. Khoze and P. Marquard, Multi-gluon collinear limits from MHV diagrams, JHEP 05 (2005) 013 [hep-ph/0503063] [INSPIRE].

[21] Z. Kunszt and D.E. Soper, Calculation of jet cross-sections in hadron collisions at order $\alpha_{S}^{3}$, Phys. Rev. D 46 (1992) 192 [InSPIRE].

[22] S. Frixione, Z. Kunszt and A. Signer, Three jet cross-sections to next-to-leading order, Nucl. Phys. B 467 (1996) 399 [hep-ph/9512328] [INSPIRE]. 
[23] S. Catani and M. Seymour, A General algorithm for calculating jet cross-sections in NLO QCD, Nucl. Phys. B 485 (1997) 291 [Erratum ibid. B 510 (1998) 503] [hep-ph/9605323] [INSPIRE].

[24] D.A. Kosower, Antenna factorization of gauge theory amplitudes, Phys. Rev. D 57 (1998) 5410 [hep-ph/9710213] [INSPIRE].

[25] D.A. Kosower, Antenna factorization in strongly ordered limits, Phys. Rev. D 71 (2005) 045016 [hep-ph/0311272] [INSPIRE].

[26] J.M. Campbell, M. Cullen and E.W.N. Glover, Four jet event shapes in electron-positron annihilation, Eur. Phys. J. C 9 (1999) 245 [hep-ph/9809429] [INSPIRE].

[27] G. Somogyi, Subtraction with hadronic initial states at NLO: An NNLO-compatible scheme, JHEP 05 (2009) 016 [arXiv:0903.1218] [INSPIRE].

[28] D.A. Kosower, Multiple singular emission in gauge theories, Phys. Rev. D 67 (2003) 116003 [hep-ph/0212097] [INSPIRE].

[29] S. Weinzierl, Subtraction terms at NNLO, JHEP 03 (2003) 062 [hep-ph/0302180] [INSPIRE].

[30] W.B. Kilgore, Subtraction terms for hadronic production processes at next-to-next-to-leading order, Phys. Rev. D 70 (2004) 031501 [hep-ph/0403128] [INSPIRE].

[31] S. Frixione and M. Grazzini, Subtraction at NNLO, JHEP 06 (2005) 010 [hep-ph/0411399] [INSPIRE].

[32] G. Somogyi, Z. Trócsányi and V. Del Duca, Matching of singly- and doubly-unresolved limits of tree-level QCD squared matrix elements, JHEP 06 (2005) 024 [hep-ph/0502226] [INSPIRE].

[33] G. Somogyi, Z. Trócsányi and V. Del Duca, A Subtraction scheme for computing QCD jet cross sections at NNLO: Regularization of doubly-real emissions, JHEP 01 (2007) 070 [hep-ph/0609042] [INSPIRE].

[34] G. Somogyi and Z. Trócsányi, A Subtraction scheme for computing QCD jet cross sections at NNLO: Regularization of real-virtual emission, JHEP 01 (2007) 052 [hep-ph/0609043] [INSPIRE].

[35] G. Somogyi and Z. Trócsányi, A Subtraction scheme for computing QCD jet cross sections at NNLO: Integrating the subtraction terms. I., JHEP 08 (2008) 042 [arXiv:0807.0509] [INSPIRE].

[36] U. Aglietti, V. Del Duca, C. Duhr, G. Somogyi and Z. Trócsányi, Analytic integration of real-virtual counterterms in NNLO jet cross sections. I., JHEP 09 (2008) 107 [arXiv: 0807.0514] [INSPIRE].

[37] P. Bolzoni, S.-O. Moch, G. Somogyi and Z. Trócsányi, Analytic integration of real-virtual counterterms in NNLO jet cross sections. II., JHEP 08 (2009) 079 [arXiv:0905.4390] [INSPIRE].

[38] P. Bolzoni, G. Somogyi and Z. Trócsányi, A subtraction scheme for computing QCD jet cross sections at NNLO: integrating the iterated singly-unresolved subtraction terms, JHEP 01 (2011) 059 [arXiv: 1011.1909] [inSPIRE].

[39] S. Catani and M. Grazzini, An NNLO subtraction formalism in hadron collisions and its application to Higgs boson production at the LHC, Phys. Rev. Lett. 98 (2007) 222002 [hep-ph/0703012] [INSPIRE]. 
[40] M. Czakon, A novel subtraction scheme for double-real radiation at NNLO, Phys. Lett. B 693 (2010) 259 [arXiv: 1005.0274] [InSPIRE].

[41] M. Czakon, Double-real radiation in hadronic top quark pair production as a proof of a certain concept, Nucl. Phys. B 849 (2011) 250 [arXiv:1101.0642] [INSPIRE].

[42] I. Bierenbaum, M. Czakon and A. Mitov, The singular behavior of one-loop massive QCD amplitudes with one external soft gluon, Nucl. Phys. B 856 (2012) 228 [arXiv:1107.4384] [INSPIRE].

[43] C. Anastasiou, F. Herzog and A. Lazopoulos, On the factorization of overlapping singularities at NNLO, JHEP 03 (2011) 038 [arXiv:1011.4867] [INSPIRE].

[44] C. Anastasiou, F. Herzog and A. Lazopoulos, The fully differential decay rate of a Higgs boson to bottom-quarks at NNLO in QCD, JHEP 03 (2012) 035 [arXiv:1110.2368] [INSPIRE].

[45] S. Bühler, F. Herzog, A. Lazopoulos and R. Müller, The fully differential hadronic production of a Higgs boson via bottom quark fusion at NNLO, JHEP 07 (2012) 115 [arXiv:1204.4415] [INSPIRE].

[46] A. Gehrmann-De Ridder, T. Gehrmann and E.W.N. Glover, Antenna subtraction at NNLO, JHEP 09 (2005) 056 [hep-ph/0505111] [INSPIRE].

[47] A. Gehrmann-De Ridder, T. Gehrmann and E.W.N. Glover, Infrared structure of $e^{+} e^{-} \rightarrow 2$ jets at NNLO, Nucl. Phys. B 691 (2004) 195 [hep-ph/0403057] [INSPIRE].

[48] A. Gehrmann-De Ridder, T. Gehrmann and E.W.N. Glover, Quark-gluon antenna functions from neutralino decay, Phys. Lett. B 612 (2005) 36 [hep-ph/0501291] [INSPIRE].

[49] A. Gehrmann-De Ridder, T. Gehrmann and E.W.N. Glover, Gluon-gluon antenna functions from Higgs boson decay, Phys. Lett. B 612 (2005) 49 [hep-ph/0502110] [INSPIRE].

[50] A. Gehrmann-De Ridder, T. Gehrmann, E.W.N. Glover and G. Heinrich, Infrared structure of $e^{+} e^{-} \rightarrow 3$ jets at NNLO, JHEP 11 (2007) 058 [arXiv:0710.0346] [INSPIRE].

[51] A. Gehrmann-De Ridder, T. Gehrmann, E.W.N. Glover and G. Heinrich, Jet rates in electron-positron annihilation at $O\left(\alpha_{s}^{3}\right)$ in QCD, Phys. Rev. Lett. 100 (2008) 172001 [arXiv:0802.0813] [INSPIRE].

[52] S. Weinzierl, NNLO corrections to 3-jet observables in electron-positron annihilation, Phys. Rev. Lett. 101 (2008) 162001 [arXiv:0807.3241] [INSPIRE].

[53] S. Weinzierl, The infrared structure of $e^{+} e^{-} \rightarrow 3$ jets at NNLO reloaded, JHEP 07 (2009) 009 [arXiv:0904.1145] [INSPIRE].

[54] S. Weinzierl, Jet algorithms in electron-positron annihilation: Perturbative higher order predictions, Eur. Phys. J. C 71 (2011) 1565 [Erratum ibid. C 71 (2011) 1717] [arXiv: 1011.6247] [INSPIRE].

[55] A. Gehrmann-De Ridder, T. Gehrmann, E.W.N. Glover and G. Heinrich, Second-order QCD corrections to the thrust distribution, Phys. Rev. Lett. 99 (2007) 132002 [arXiv:0707.1285] [INSPIRE].

[56] A. Gehrmann-De Ridder, T. Gehrmann, E.W.N. Glover and G. Heinrich, NNLO corrections to event shapes in $e^{+} e^{-}$annihilation, JHEP 12 (2007) 094 [arXiv:0711.4711] [INSPIRE].

[57] A. Gehrmann-De Ridder, T. Gehrmann, E.W.N. Glover and G. Heinrich, NNLO moments of event shapes in $e^{+} e^{-}$annihilation, JHEP 05 (2009) 106 [arXiv:0903.4658] [INSPIRE]. 
[58] S. Weinzierl, Event shapes and jet rates in electron-positron annihilation at NNLO, JHEP 06 (2009) 041 [arXiv:0904.1077] [INSPIRE].

[59] S. Weinzierl, Moments of event shapes in electron-positron annihilation at NNLO, Phys. Rev. D 80 (2009) 094018 [arXiv:0909.5056] [INSPIRE].

[60] G. Dissertori et al., First determination of the strong coupling constant using NNLO predictions for hadronic event shapes in $e^{+} e^{-}$annihilations, JHEP 02 (2008) 040 [arXiv:0712.0327] [INSPIRE].

[61] G. Dissertori et al., Determination of the strong coupling constant using matched $N N L O+N L L A$ predictions for hadronic event shapes in $e^{+} e^{-}$annihilations, JHEP 08 (2009) 036 [arXiv:0906.3436] [INSPIRE].

[62] G. Dissertori et al., Precise determination of the strong coupling constant at NNLO in QCD from the three-jet rate in electron-positron annihilation at $L E P$, Phys. Rev. Lett. 104 (2010) 072002 [arXiv:0910.4283] [INSPIRE].

[63] T. Becher and M.D. Schwartz, A precise determination of $\alpha_{s}$ from LEP thrust data using effective field theory, JHEP 07 (2008) 034 [arXiv: 0803.0342] [INSPIRE].

[64] Y.-T. Chien and M.D. Schwartz, Resummation of heavy jet mass and comparison to LEP data, JHEP 08 (2010) 058 [arXiv: 1005.1644] [InSPIRE].

[65] R. Abbate, M. Fickinger, A.H. Hoang, V. Mateu and I.W. Stewart, Thrust at $N^{3} L L$ with Power Corrections and a Precision Global Fit for $\alpha_{s}\left(m_{Z}\right)$, Phys. Rev. D 83 (2011) 074021 [arXiv: 1006.3080] [INSPIRE].

[66] R. Davison and B. Webber, Non-Perturbative Contribution to the Thrust Distribution in $e^{+} e^{-}$Annihilation, Eur. Phys. J. C 59 (2009) 13 [arXiv:0809.3326] [INSPIRE].

[67] JADE collaboration, S. Bethke, S. Kluth, C. Pahl and J. Schieck, Determination of the Strong Coupling $\alpha_{s}$ from hadronic Event Shapes with $O\left(\alpha_{s}^{3}\right)$ and resummed $Q C D$ predictions using JADE Data, Eur. Phys. J. C 64 (2009) 351 [arXiv:0810.1389] [INSPIRE].

[68] OPAL collaboration, G. Abbiendi et al., Determination of $\alpha_{s}$ using OPAL hadronic event shapes at $\sqrt{s}=91-209 \mathrm{GeV}$ and resummed NNLO calculations,

Eur. Phys. J. C 71 (2011) 1733 [arXiv:1101.1470] [INSPIRE].

[69] J. Schieck, S. Bethke, S. Kluth, C. Pahl and Z. Trócsányi, Measurement of the strong coupling $\alpha_{S}$ from the three-jet rate in $e^{+} e^{-}$-annihilation using JADE data, arXiv: 1205.3714 [INSPIRE].

[70] T. Gehrmann, M. Jaquier and G. Luisoni, Hadronization effects in event shape moments, Eur. Phys. J. C 67 (2010) 57 [arXiv:0911.2422] [InSPIRE].

[71] A. Gehrmann-De Ridder and M. Ritzmann, NLO Antenna Subtraction with Massive Fermions, JHEP 07 (2009) 041 [arXiv: 0904.3297] [INSPIRE].

[72] G. Abelof and A. Gehrmann-De Ridder, Antenna subtraction for the production of heavy particles at hadron colliders, JHEP 04 (2011) 063 [arXiv:1102.2443] [INSPIRE].

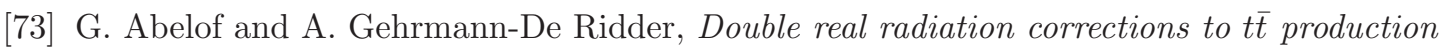
at the LHC: the all-fermion processes, JHEP 04 (2012) 076 [arXiv:1112.4736] [INSPIRE].

[74] W. Bernreuther, C. Bogner and O. Dekkers, The real radiation antenna function for $S \rightarrow Q \bar{Q} q \bar{q}$ at $N N L O Q C D, J H E P 06$ (2011) 032 [arXiv:1105.0530] [INSPIRE]. 
[75] W.T. Giele, D.A. Kosower and P.Z. Skands, A simple shower and matching algorithm, Phys. Rev. D 78 (2008) 014026 [arXiv:0707.3652] [INSPIRE].

[76] W.T. Giele, D. Kosower and P.Z. Skands, Higher-Order Corrections to Timelike Jets, Phys. Rev. D 84 (2011) 054003 [arXiv:1102.2126] [InSPIRE].

[77] A. Gehrmann-De Ridder, M. Ritzmann and P.Z. Skands, Timelike Dipole-Antenna Showers with Massive Fermions, Phys. Rev. D 85 (2012) 014013 [arXiv:1108.6172] [InSPIRE].

[78] J.J. Lopez-Villarejo and P.Z. Skands, Efficient Matrix-Element Matching with Sector Showers, JHEP 11 (2011) 150 [arXiv:1109.3608] [INSPIRE].

[79] A. Daleo, T. Gehrmann and D. Maître, Antenna subtraction with hadronic initial states, JHEP 04 (2007) 016 [hep-ph/0612257] [INSPIRE].

[80] E.W.N. Glover and J. Pires, Antenna subtraction for gluon scattering at NNLO, JHEP 06 (2010) 096 [arXiv: 1003.2824] [INSPIRE].

[81] A. Gehrmann-De Ridder, E.W.N. Glover and J. Pires, Real-Virtual corrections for gluon scattering at NNLO, JHEP 02 (2012) 141 [arXiv:1112.3613] [INSPIRE].

[82] A. Daleo, A. Gehrmann-De Ridder, T. Gehrmann and G. Luisoni, Antenna subtraction at NNLO with hadronic initial states: initial-final configurations, JHEP 01 (2010) 118 [arXiv: 0912.0374] [INSPIRE].

[83] T. Gehrmann and P.F. Monni, Antenna subtraction at NNLO with hadronic initial states: real-virtual initial-initial configurations, JHEP 12 (2011) 049 [arXiv:1107.4037] [INSPIRE].

[84] R. Boughezal, A. Gehrmann-De Ridder and M. Ritzmann, Antenna subtraction at NNLO with hadronic initial states: double real radiation for initial-initial configurations with two quark flavours, JHEP 02 (2011) 098 [arXiv: 1011.6631] [INSPIRE].

[85] T. Binoth and G. Heinrich, An automatized algorithm to compute infrared divergent multiloop integrals, Nucl. Phys. B 585 (2000) 741 [hep-ph/0004013] [INSPIRE].

[86] G. Heinrich, A numerical method for NNLO calculations, Nucl. Phys. Proc. Suppl. 116 (2003) 368 [hep-ph/0211144] [inSPIRE].

[87] C. Anastasiou, K. Melnikov and F. Petriello, A new method for real radiation at NNLO, Phys. Rev. D 69 (2004) 076010 [hep-ph/0311311] [inSPIRE].

[88] T. Binoth and G. Heinrich, Numerical evaluation of phase space integrals by sector decomposition, Nucl. Phys. B 693 (2004) 134 [hep-ph/0402265] [INSPIRE].

[89] C. Anastasiou, K. Melnikov and F. Petriello, Higgs boson production at hadron colliders: Differential cross sections through next-to-next-to-leading order, Phys. Rev. Lett. 93 (2004) 262002 [hep-ph/0409088] [INSPIRE].

[90] C. Anastasiou, K. Melnikov and F. Petriello, Fully differential Higgs boson production and the di-photon signal through next-to-next-to-leading order, Nucl. Phys. B 724 (2005) 197 [hep-ph/0501130] [INSPIRE].

[91] C. Anastasiou, G. Dissertori and F. Stoöckli, NNLO QCD predictions for the $H \rightarrow W W \rightarrow \ell \nu \ell \nu$ signal at the LHC, JHEP 09 (2007) 018 [arXiv:0707.2373] [INSPIRE].

[92] K. Melnikov and F. Petriello, The $W$ boson production cross section at the LHC through $O\left(\alpha_{s}^{2}\right)$, Phys. Rev. Lett. 96 (2006) 231803 [hep-ph/0603182] [INSPIRE].

[93] M. Grazzini, NNLO predictions for the Higgs boson signal in the $H \rightarrow W W \rightarrow \ell \nu \ell \nu$ and $H \rightarrow Z Z \rightarrow 4 \ell$ decay channels, JHEP 02 (2008) 043 [arXiv:0801.3232] [INSPIRE]. 
[94] S. Catani, L. Cieri, G. Ferrera, D. de Florian and M. Grazzini, Vector boson production at hadron colliders: A fully exclusive QCD calculation at NNLO,

Phys. Rev. Lett. 103 (2009) 082001 [arXiv:0903.2120] [InSPIRE].

[95] S. Catani, G. Ferrera and M. Grazzini, W Boson Production at Hadron Colliders: The Lepton Charge Asymmetry in NNLO QCD, JHEP 05 (2010) 006 [arXiv:1002.3115] [INSPIRE].

[96] G. Ferrera, M. Grazzini and F. Tramontano, Associated WH production at hadron colliders: a fully exclusive QCD calculation at NNLO, Phys. Rev. Lett. 107 (2011) 152003 [arXiv:1107.1164] [INSPIRE].

[97] S. Catani, L. Cieri, D. de Florian, G. Ferrera and M. Grazzini, Diphoton production at hadron colliders: a fully-differential QCD calculation at $N N L O$,

Phys. Rev. Lett. 108 (2012) 072001 [arXiv:1110.2375] [InSPIRE].

[98] P. Bärnreuther, M. Czakon and A. Mitov, Percent Level Precision Physics at the Tevatron: First Genuine NNLO QCD Corrections to $q \bar{q} \rightarrow t \bar{t}+X$, arXiv:1204.5201 [INSPIRE].

[99] M. Czakon and A. Mitov, NNLO corrections to top-pair production at hadron colliders: the all-fermionic scattering channels, arXiv:1207.0236 [INSPIRE].

[100] J. Vermaseren, New features of FORM, math-ph/0010025 [INSPIRE].

[101] J. Kuipers, T. Ueda, J. Vermaseren and J. Vollinga, FORM version 4.0, arXiv:1203.6543 [INSPIRE].

[102] D. de Florian and M. Grazzini, The Structure of large logarithmic corrections at small transverse momentum in hadronic collisions, Nucl. Phys. B 616 (2001) 247 [hep-ph/0108273] [INSPIRE].

[103] C. Anastasiou, L.J. Dixon, K. Melnikov and F. Petriello, High precision QCD at hadron colliders: Electroweak gauge boson rapidity distributions at NNLO, Phys. Rev. D 69 (2004) 094008 [hep-ph/0312266] [INSPIRE].

[104] C. Anastasiou and K. Melnikov, Higgs boson production at hadron colliders in NNLO QCD, Nucl. Phys. B 646 (2002) 220 [hep-ph/0207004] [INSPIRE].

[105] K. Chetyrkin and F. Tkachov, Integration by Parts: The Algorithm to Calculate $\beta$-functions in 4 Loops, Nucl. Phys. B 192 (1981) 159 [INSPIRE].

[106] S. Laporta, High precision calculation of multiloop Feynman integrals by difference equations, Int. J. Mod. Phys. A 15 (2000) 5087 [hep-ph/0102033] [InSPIRE].

[107] A. Smirnov, Algorithm FIRE - Feynman Integral REduction, JHEP 10 (2008) 107 [arXiv:0807.3243] [INSPIRE].

[108] P.F. Monni, T. Gehrmann and G. Luisoni, Two-Loop Soft Corrections and Resummation of the Thrust Distribution in the Dijet Region, JHEP 08 (2011) 010 [arXiv:1105.4560] [INSPIRE].

[109] T. Huber and D. Maître, HypExp: A Mathematica package for expanding hypergeometric functions around integer-valued parameters, Comput. Phys. Commun. 175 (2006) 122 [hep-ph/0507094] [INSPIRE].

[110] T. Huber and D. Maître, HypExp 2, Expanding Hypergeometric Functions about Half-Integer Parameters, Comput. Phys. Commun. 178 (2008) 755 [arXiv:0708.2443] [INSPIRE]. 
[111] E. Remiddi and J. Vermaseren, Harmonic polylogarithms, Int. J. Mod. Phys. A 15 (2000) 725 [hep-ph/9905237] [INSPIRE].

[112] C. Anastasiou, E.W.N. Glover, C. Oleari and M. Tejeda-Yeomans, Two-loop QCD corrections to the scattering of massless distinct quarks, Nucl. Phys. B 601 (2001) 318 [hep-ph/0010212] [INSPIRE].

[113] E.W.N. Glover, C. Oleari and M. Tejeda-Yeomans, Two loop QCD corrections to gluon-gluon scattering, Nucl. Phys. B 605 (2001) 467 [hep-ph/0102201] [INSPIRE].

[114] C. Anastasiou, E.W.N. Glover and M. Tejeda-Yeomans, Two loop QED and QCD corrections to massless fermion boson scattering, Nucl. Phys. B 629 (2002) 255 [hep-ph/0201274] [INSPIRE].

[115] E.W.N. Glover and M. Tejeda-Yeomans, Two loop QCD helicity amplitudes for massless quark massless gauge boson scattering, JHEP 06 (2003) 033 [hep-ph/0304169] [INSPIRE].

[116] E.W.N. Glover, Two loop QCD helicity amplitudes for massless quark quark scattering, JHEP 04 (2004) 021 [hep-ph/0401119] [INSPIRE].

[117] Z. Bern, A. De Freitas and L.J. Dixon, Two loop amplitudes for gluon fusion into two photons, JHEP 09 (2001) 037 [hep-ph/0109078] [INSPIRE].

[118] Z. Bern, A. De Freitas and L.J. Dixon, Two loop helicity amplitudes for gluon-gluon scattering in QCD and supersymmetric Yang-Mills theory, JHEP 03 (2002) 018 [hep-ph/0201161] [INSPIRE].

[119] Z. Bern, A. De Freitas and L.J. Dixon, Two loop helicity amplitudes for quark gluon scattering in QCD and gluino gluon scattering in supersymmetric Yang-Mills theory, JHEP 06 (2003) 028 [hep-ph/0304168] [INSPIRE].

[120] A. De Freitas and Z. Bern, Two-loop helicity amplitudes for quark-quark scattering in QCD and gluino-gluino scattering in supersymmetric Yang-Mills theory, JHEP 09 (2004) 039 [hep-ph/0409007] [INSPIRE].

[121] L. Garland, T. Gehrmann, E.W.N. Glover, A. Koukoutsakis and E. Remiddi, The Two loop QCD matrix element for $e^{+} e^{-} \rightarrow 3$ jets, Nucl. Phys. B 627 (2002) 107 [hep-ph/0112081] [INSPIRE].

[122] L. Garland, T. Gehrmann, E.W.N. Glover, A. Koukoutsakis and E. Remiddi, Two loop QCD helicity amplitudes for $e^{+} e^{-} \rightarrow 3$ jets, Nucl. Phys. B 642 (2002) 227 [hep-ph/0206067] [INSPIRE].

[123] T. Gehrmann and L. Tancredi, Two-loop QCD helicity amplitudes for $q \bar{q} \rightarrow W^{ \pm} \gamma$ and $q \bar{q} \rightarrow Z^{0} \gamma$, JHEP 02 (2012) 004 [arXiv: 1112.1531] [INSPIRE].

[124] T. Gehrmann, M. Jaquier, E.W.N. Glover and A. Koukoutsakis, Two-Loop QCD Corrections to the Helicity Amplitudes for $H \rightarrow 3$ partons, JHEP 02 (2012) 056 [arXiv:1112.3554] [INSPIRE].

[125] J. Currie, Antenna Subtraction in $p Q C D$ at NNLO, arXiv:1111.4810 [INSPIRE].

[126] A. Gehrmann-De Ridder, T. Gehrmann, E.W.N. Glover and J. Pires, Double virtual corrections for gluon scattering at $N N L O$, in preparation. 